\title{
A HILBERT SPACE OF DIRICHLET SERIES AND SYSTEMS OF DILATED FUNCTIONS IN $L^{2}(0,1)$
}

\author{
HÅkan Hedenmalm, Peter Lindqvist, and Kristian SeiP
}

\begin{abstract}
For a function $\varphi$ in $L^{2}(0,1)$, extended to the whole real line as an odd periodic function of period 2 , we ask when the collection of dilates $\varphi(n x), n=$ $1,2,3, \ldots$, constitutes a Riesz basis or a complete sequence in $L^{2}(0,1)$. The problem translates into a question concerning multipliers and cyclic vectors in the Hilbert space $\mathcal{H}$ of Dirichlet series $f(s)=\sum_{n} a_{n} n^{-s}$, where the coefficients $a_{n}$ are square summable. It proves useful to model $\mathcal{H}$ as the $H^{2}$ space of the infinite-dimensional polydisk, or, which is the same, the $H^{2}$ space of the character space, where a character is a multiplicative homomorphism from the positive integers to the unit circle. For given $f$ in $\mathcal{H}$ and characters $\chi, f_{\chi}(s)=\sum_{n} a_{n} \chi(n) n^{-s}$ is a vertical limit function of $f$. We study certain probabilistic properties of these vertical limit functions.
\end{abstract}

\section{INTRODUCTION}

The purpose of this paper is twofold. First, we study systems of functions of the form $\varphi(x), \varphi(2 x), \varphi(3 x), \ldots$, and second, we consider the Hardy space $H^{2}$ of the infinite-dimensional polydisk. Building on ideas of Arne Beurling and Harald Bohr, we find that the two topics are intimately connected, the common feature being the use of Dirichlet series.

Let $\varphi \in L^{2}(0,1)$ be given and consider $\varphi$ as defined on the whole real axis by extending it to an odd periodic function of period 2. The Riesz-Fischer theorem of Fourier analysis states that for $\varphi(x)=\sqrt{2} \sin (\pi x)$ the sequence $\varphi(n x), n=$ $1,2,3, \ldots$, is an orthonormal basis in the Hilbert space $L^{2}(0,1)$. The question raised in this paper is which functions can take the place of the sine in this theorem. It is clear that the statement must be weakened, because the only orthogonal bases are obtained from $\varphi(x)=C \sin (\pi x)$. If we instead ask for a classification of those $\varphi$ for which the system $\{\varphi(n x)\}_{n}$ is a Riesz basis (a basis orthonormal with respect to an equivalent norm) or of those $\varphi$ for which the same system is a complete sequence in $L^{2}(0,1)$, we are led to profound problems.

The latter of the two problems - the completeness problem - was stated by Beurling in his seminar on harmonic analysis in Uppsala in 1945. A brief note from this seminar is found in [1]. Beurling's note indicates that a natural way to

The work of the first author was supported in part by the Swedish Natural Science Research Council (NFR), and that of the second and third authors by the Norwegian Research Council (NFR). While at MSRI, Berkeley, the first and third authors were also supported in part by NSF grant DMS-9022140. 
approach these problems is to associate to the given function

$$
\varphi(x)=\sum_{n=1}^{\infty} a_{n} \sqrt{2} \sin (n \pi x)
$$

the Dirichlet series

$$
S \varphi(s)=\sum_{n=1}^{\infty} a_{n} n^{-s},
$$

and to try to express the Riesz basis and completeness properties in terms of analytic properties of $S \varphi(s)$. This approach has proved fruitful. We have solved completely the Riesz basis problem (Theorems 3.1 and 5.2 below): the system $\{\varphi(n x)\}_{n}$ is a Riesz basis in $L^{2}(0,1)$ if and only if (1-1) defines an analytic function bounded away from zero and infinity in the half-plane $\Re s>0$.

A major part of this paper consists of a study of Dirichlet series of the form (1-1) with $\sum_{n}\left|a_{n}\right|^{2}<+\infty$. Such Dirichlet series form, in a natural way, a Hilbert space of analytic functions on the half-plane $\Re s>\frac{1}{2}$. From now on, we denote this space by $\mathcal{H}$. In Sections $2-4$, we seek to reveal the basic analytic features of this space. A central problem is to characterize the so-called multipliers of $\mathcal{H}$. Theorem 3.1 below states that the multipliers are precisely the bounded analytic functions in the right half-plane $\Re s>0$ which can be represented as Dirichlet series. This result is crucial for the characterization of the Riesz bases $\{\varphi(n x)\}_{n}$ mentioned above. Apart for its relevance for the dilation Riesz basis problem, the study of the space $\mathcal{H}$ can also be motivated by the mere fact that its kernel function $K_{\mathcal{H}}(z, w)$ is more or less the Riemann zeta function: $K_{\mathcal{H}}(z, w)=\zeta(z+\bar{w})$.

Following an idea of Bohr, we find it both convenient and illuminating to use the infinite-dimensional polydisk for the study of $\mathcal{H}$. This leads us to power series in infinitely many variables, a concept studied already by Hilbert, and to an identification of $\mathcal{H}$ as the Hardy space $H^{2}$ of the infinite-dimensional polydisk. The infinitedimensional polycircle can be identified with the group $\Xi$ of complex-valued characters $\chi$ on the positive integers, which satisfy $|\chi(n)|=1$ and $\chi(m n)=\chi(m) \chi(n)$. The characters constitute the (compact) dual group of the discrete multiplicative group of positive rationals $\left\langle\mathbb{Q}_{+}, \cdot\right\rangle$.

A function $f \in \mathcal{H}$ is said to be cyclic provided that the collection of functions $f g$, where $g$ is a finite Dirichlet series, is dense in $\mathcal{H}$. A basic observation is that the system $\left\{\varphi_{n}\right\}_{n}$ is complete if and only if the Dirichlet series $S \varphi$ is cyclic in $\mathcal{H}$. We restate cyclicity in terms of our model of $\mathcal{H}$ as the $H^{2}$ space of the infinitedimensional polydisk, and state some conditions for cyclicity. There is some overlap between these conditions and results due to Henry Helson [16], who studied cyclicity in a more general context.

Since the multipliers of $\mathcal{H}$ extend analytically and boundedly to $\Re s>0$, but the functions of $\mathcal{H}$ need only be analytic on $\Re s>\frac{1}{2}$, one is led to suspect that, nevertheless, in a sense to be made precise, a function in $\mathcal{H}$, picked at random, almost surely extends holomorphically to $\Re s>0$. Given a Dirichlet series in $\mathcal{H}$,

$$
f(s)=\sum_{n=1}^{\infty} a_{n} n^{-s},
$$

we consider, for characters $\chi$,

$$
f_{\chi}(s)=\sum^{\infty} a_{n} \chi(n) n^{-s}
$$


which again is an element of $\mathcal{H}$. The functions $f_{\chi}$ are the normal limits of vertical translates of $f$. Almost every one of them has a convergent Dirichlet series on $\Re s>0$, and is hence holomorphic there. The "almost every" is with respect to the Haar measure on the character group $\Xi$, which is normalized to have total mass 1. This result was obtained in a more abstract setting by Helson [16]. A curious consequence is that a probabilistic "Riemann hypothesis" (Corollary 4.8) holds: for almost all characters $\chi$, the Dirichlet series $\zeta_{\chi}(s)=\sum_{n=1}^{\infty} \chi(n) n^{-s}$ converges to a zero-free holomorphic function in the region $\Re s>\frac{1}{2}$. The convergence part has the following interpretation: for almost all characters $\chi$,

$$
\sum_{n=1}^{N} \chi(n)=O\left(N^{1 / 2+\varepsilon}\right) \quad \text { as } \quad N \rightarrow+\infty
$$

holds for fixed $\varepsilon>0$. For each $n$, it is fruitful to regard the function $\chi(n)$ as a stochastic variable, which for $n>1$ is uniformly distributed on $\mathbb{T}$. The stochastic variables $\chi(p)$, where $p$ runs through the primes, are then mutually independent, and the mutual dependence of the $\chi(n)$, as $n$ runs through the positive integers, is governed by the multiplicative rule $\chi(m n)=\chi(m) \chi(n)$. The above estimate of the partial sum $\sum_{n: n<N} \chi(n)$ is what one would expect if all the $\chi(n)$ were mutually independent, so although they do satisfy complicated multiplicative dependence relations, this is insignificant additively.

The above-mentioned assertions may be compared with Jean-Pierre Kahane's paper $[\mathbf{1 8}]$ on random Dirichlet series. Kahane works with functions $f_{\chi}(s)$, where $\chi(n)$ is treated as a sequence of independent random variables, and hence no multiplicativity property holds.

In [15], Helson suggests that the classical theory of Dirichlet series be combined with modern techniques from harmonic and functional analysis. We hope that the present paper can inspire work in that direction.

\section{The Hilbert spaces $\mathcal{H}$ And $H^{2}\left(\mathbb{D}^{\infty}\right)$}

2.1 The space $\mathcal{H}$ and preliminaries on Dirichlet series. We are concerned with Dirichlet series of the form

$$
f(s)=\sum_{n=1}^{\infty} a_{n} n^{-s}
$$

where $s=\sigma+i t$ is a complex variable. We recall briefly some classical facts about such series. There are a number of critical lines or abscissæ connected with (2-1). We have the abscissa of absolute convergence $\sigma_{a}$ and the abscissa of ordinary convergence $\sigma_{c}$. These numbers are such that the series converges in the prescribed sense to the right but not to the left of the abscissa in question. We also have the abscissa of uniform convergence $\sigma_{u}$, defined as the infimum of those $\sigma_{0}$ for which the series converges uniformly in the half-plane $\Re s>\sigma_{0}$. We have trivially $-\infty \leq \sigma_{c} \leq \sigma_{u} \leq \sigma_{a} \leq+\infty$ and $\sigma_{a}-\sigma_{c} \leq 1$ if anyone of the abscissæ is finite. A theorem of Bohr [4] says that $\sigma_{a}-\sigma_{u} \leq \frac{1}{2}$, and this inequality is sharp, as was shown by Bohnenblust and Hille [2].

When we need to indicate which function we are taking the abscissæ of, we 
for the abscissa of convergence. In terms of the partial sums of the coefficients, $S_{N}=\sum_{n: n<N} a_{n}$, the abscissa of convergence of the Dirichlet series (2-1) can be expressed as follows: unless $\sigma_{c}(f)$ is negative, $\sigma_{c}(f)$ equals the infimum of all positive real numbers $\alpha$ for which $S_{N}=O\left(N^{\alpha}\right)$ as $N \rightarrow+\infty$.

Of relevance to us is also the abscissa of regularity and boundedness $\sigma_{b}$, which is the infimum of those $\sigma_{0}$ for which the function defined by (2-1) (possibly by analytic continuation from a smaller half-plane) is analytic and bounded for $\Re s>\sigma_{0}$. We shall need the following result of Bohr [3].

Lemma 2.1. (Bohr's theorem) $\sigma_{u}=\sigma_{b}$.

For a more complete account of the basic facts on the convergence of Dirichlet series, the reader is referred to [12] and Bohr's thesis [5].

We will assume that the function $f$ given by the Dirichlet series (2-1) belongs to $\mathcal{H}$, that is,

$$
\sum_{n=1}^{\infty}\left|a_{n}\right|^{2}<+\infty
$$

The Cauchy-Schwarz inequality yields

$$
\left(\sum_{n=1}^{\infty}\left|a_{n} n^{-s}\right|\right)^{2} \leq \sum_{n=1}^{\infty}\left|a_{n}\right|^{2} \sum_{n=1}^{\infty} n^{-2 \sigma}, \quad s=\sigma+i t
$$

and so the abscissa of absolute convergence is at most $\frac{1}{2}$ for the series $(2-1)$. The abscissa of convergence may equal $\frac{1}{2}$, as is seen by the example $a_{n}=\left(n^{1 / 2} \log (n+\right.$ $1))^{-1}$.

As mentioned in the introduction, the collection of Dirichlet series (2-1) satisfying $(2-2)$ is denoted by $\mathcal{H}$. It is a complex Hilbert space when endowed with the inner product

$$
\langle f, g\rangle_{\mathcal{H}}=\sum_{n=1}^{\infty} a_{n} \bar{b}_{n}
$$

where $f(s)=\sum_{n} a_{n} n^{-s}$, and $g(s)=\sum_{n} b_{n} n^{-s}$. Thus, formally, $\mathcal{H}$ is just $l^{2}(\mathbb{N})$, where $\mathbb{N}=\{1,2,3, \ldots\}$ is the collection of natural numbers. However, as a Hilbert space of analytic functions in the half-plane $\Re s>\frac{1}{2}, \mathcal{H}$ has a rich and interesting structure. To be specific, let us mention the classical problem of multiplication of two Dirichlet series. Formally, the product of $f(s)=\sum_{n} a_{n} n^{-s}$ and $g(s)=$ $\sum_{n} b_{n} n^{-s}$ is again a Dirichlet series

$$
f(s) g(s)=\sum_{n} c_{n} n^{-s}
$$

with

$$
c_{n}=\sum_{k, l: k l=n} a_{k} b_{l}
$$

For $f \in \mathcal{H}$ and $g \in \mathcal{H}$, the product $f g$ need not be in $\mathcal{H}$, although the abscissa of absolute convergence of (2-3) can be shown to be at most $\frac{1}{2}$ (as it is for functions in $\mathcal{H})$. We are lead to the multiplier problem: Find those functions $m$, analytic in $\Re s>\frac{1}{2}$, for which $m(s) f(s)$ is in $\mathcal{H}$ for every $f \in \mathcal{H}$. The collection of these 
Let us finally mention a classical theorem which we will refer to from time to time. We say that a set of real numbers $\xi_{1}, \xi_{2}, \ldots, \xi_{r}$ is $\mathbb{Q}$-linearly independent if

$$
c_{1} \xi_{1}+c_{2} \xi_{2}+\cdots+c_{r} \xi_{r}=0
$$

holds with integer coefficients $c_{1}, c_{2}, \ldots, c_{r}$ only when the coefficients are all zero. By the fundamental theorem of arithmetic, the prime example of a $\mathbb{Q}$-linearly independent set of numbers is the image under the logarithm function of any finite set of different primes. We have the fundamental

Lemma 2.2. (Kronecker's theorem) Suppose the real numbers $1, \vartheta_{1}, \vartheta_{2}, \ldots, \vartheta_{k}$ are $\mathbb{Q}$-linearly independent. Let $\alpha_{1}, \alpha_{2}, \ldots, \alpha_{k}$ be arbitrary real numbers, and $N$ and $\epsilon$ be given positive. Then there are integers

$$
n>N, \quad q_{1}, q_{2}, \ldots, q_{k}
$$

such that

$$
\left|n \vartheta_{m}-q_{m}-\alpha_{m}\right|<\epsilon \quad(m=1,2, \ldots, k) .
$$

For a proof, we refer to Chapter XXIII of [13].

To give an example of how Kronecker's theorem applies to our context, we mention that an immediate consequence is the following identity:

$$
\left\|1+\sum_{p} a_{p} p^{-s}\right\|_{\infty}=1+\sum_{p}\left|a_{p}\right|
$$

here the sum is only over prime indices, and

$$
\|f\|_{\infty}=\sup _{\Re s>0}|f(s)|
$$

2.2. The Hardy space $H^{2}$ of the infinite-dimensional polydisk. Let $f$ be the Dirichlet series (2-1), and suppose $f \in \mathcal{H}$. A fundamental observation, first made by Bohr $[4]$, is that if we put

$$
z_{1}=2^{-s}, z_{2}=3^{-s}, \ldots, z_{m}=p_{m}^{-s}, \ldots
$$

where $p_{m}$ denotes the $m$-th prime, then, in view of the fundamental theorem of arithmetic, the Dirichlet series (2-1) can be considered as a power series of infinitely many variables. The point is, as Bohr clarifies in his work, that the quantities $z_{m}=p_{m}^{-s}, m=1,2,3, \ldots$, "obwohl sie ja in Wirklichkeit Funktionen nur des einen Parameters $s$ sind, sich in mancher Beziehung fast ganz benehmen, als wären sie von einander unabhängige Variable." We put $z=\left(z_{m}\right)_{m}=\left(z_{1}, z_{2}, z_{3}, \ldots\right)$, and write

$$
n=p_{k_{1}}^{\nu_{1}} p_{k_{2}}^{\nu_{2}} \ldots p_{k_{r}}^{\nu_{r}}
$$

so that (2-1) formally takes the form

$$
\mathfrak{Q} f(z)=\sum^{\infty} a_{n} z_{k_{1}}^{\nu_{1}} \cdot z_{k_{2}}^{\nu_{2}} \cdots z_{k_{r}}^{\nu_{r}} .
$$


From now on, for a given element $f \in \mathcal{H}, \mathfrak{Q} f$ denotes the corresponding power series, and we drop the relationship between $z$ and $s$.

Another, sometimes more convenient, way to think of the extension $\mathfrak{Q} f$ of $f$ in $(2-5)$ is to write it as

$$
\mathfrak{Q} f(\phi)=\sum_{n=1}^{\infty} a_{n} \phi(n),
$$

where $\phi$ is a quasi-character, by which we mean a mapping $\phi: \mathbb{N} \rightarrow \mathbb{C}$, which is multiplicative, $\phi(m n)=\phi(m) \phi(n)$, and has $\phi(1)=1$ and $\phi(n) \in \mathbb{D}$ for $n>1$. Here, $\mathbb{D}$ is the open unit disk. If we put, for the $m$-th prime $p_{m}, \phi\left(p_{m}\right)=z_{m}$, and identify $\phi$ with $z=\left(z_{m}\right)_{m}$, then (2-5) and (2-5') express the same function $\mathfrak{Q} f$. The Cauchy-Schwarz inequality applied to (2-5) (or (2-5')) and Euler's product formula give

$$
\begin{aligned}
|\mathfrak{Q} f(z)|^{2}=|\mathfrak{Q} f(\phi)|^{2} \leq & \sum_{n=1}^{\infty}\left|a_{n}\right|^{2} \sum_{n=1}^{\infty}|\phi(n)|^{2} \\
& =\|f\|_{\mathcal{H}}^{2} \cdot \prod_{p}\left(1-|\phi(p)|^{2}\right)^{-1}=\|f\|_{\mathcal{H}}^{2} \cdot \prod_{m=1}^{\infty}\left(1-\left|z_{m}\right|^{2}\right)^{-1},
\end{aligned}
$$

provided that $\left|z_{m}\right|<1$ for every $m$. It follows that we have bounded point evaluation at $z$, id est,

$$
|\mathfrak{Q} f(z)| \leq C(z)\|f\|_{\mathcal{H}}
$$

if and only if $\left|z_{m}\right|<1$ for every $m$ and

$$
\sum_{m=1}^{\infty}\left|z_{m}\right|^{2}<+\infty
$$

We denote by $\mathbb{D}^{\infty}$ the set of $z=\left(z_{m}\right)_{m=1}^{\infty}$ for which $\left|z_{m}\right|<1$ for every $m$, and call it the infinite-dimensional polydisk. The collection of power series (2-5) with $f \in \mathcal{H}$ can be regarded as a space of analytic functions on $\mathbb{D}^{\infty} \cap l^{2}(\mathbb{N})$. We denote this space by $H^{2}\left(\mathbb{D}^{\infty}\right)$, and supply it with the Hilbert space structure induced by $\mathcal{H}$. Note that $H^{2}\left(\mathbb{D}^{\infty}\right)$ differs from $\mathcal{H}$ only in that its elements are considered as functions on $\mathbb{D}^{\infty} \cap l^{2}(\mathbb{N})$ rather than on the half-plane $\Re s>\frac{1}{2}$. In other words, $\mathcal{H}$ results from considering (2-5') for the special quasi-characters $\phi_{s}(n)=n^{-s}$.

Before discussing further the nature of $H^{2}\left(\mathbb{D}^{\infty}\right)$, we mention an interesting problem, treated already by Hilbert [17]: Is it possible to extend the meaning of (2-5) beyond the set $\mathbb{D}^{\infty} \cap l^{2}(\mathbb{N})$, at least under certain favorable cirumstances? As indicated by Bohr, Hilbert's solution to this problem has a remarkable significance for Dirichlet series [4]. Let

$$
\Omega=\left\{z=\left(z_{m}\right)_{m} \in \mathbb{D}^{\infty}:\left|z_{m}\right|<\beta_{m} \text { for every } m\right\}
$$

where $\left\{\beta_{m}\right\}_{m}$ is a sequence of positive numbers, $0<\beta_{m} \leq 1$. For a given point $z=\left(z_{1}, z_{2}, \ldots\right) \in \mathbb{D}^{\infty}$, we put 
id est, the $j$-th coordinate for $j>m$ is put equal to zero. For the time being, let $f$ be a Dirichlet series (2-1), about which we assume only that it converges on some half-plane $\Re s>\sigma_{c}$ (we do not require (2-2) to hold). The formal power series $\mathfrak{Q} f\left(z^{(m)}\right)$ is the " $m$-te Abschnitt" of $\mathfrak{Q} f$ at $z$, and it only depends on the finitely many coordinates $z_{1}, \ldots, z_{m}$. If the " $m$-te Abschnitt" converges absolutely on $\Omega$ for each $m$, and there exists a constant $C=C(f)$ such that

$$
\left|\mathfrak{Q} f\left(z^{(m)}\right)\right| \leq C, \quad z \in \Omega
$$

independently of $m, \mathfrak{Q} f$ is said to be finitely bounded in $\Omega$. In particular, the collection of series $\mathfrak{Q} f$ that are finitely bounded in $\mathbb{D}^{\infty}$ is denoted by $H^{\infty}\left(\mathbb{D}^{\infty}\right)$, and we write

$$
\|\mathfrak{Q} f\|_{H^{\infty}\left(\mathbb{D}^{\infty}\right)}=\sup _{m, z}\left|\mathfrak{Q} f\left(z^{(m)}\right)\right|
$$

where $z$ ranges over $\mathbb{D}^{\infty}$. It is natural to define the value of $\mathfrak{Q} f(z)$ as the limit of $\mathfrak{Q} f\left(z^{(m)}\right)$ as $m \rightarrow+\infty$. The limit makes sense for $z \in \mathbb{D}^{\infty} \cap c_{0}(\mathbb{N})$, where $c_{0}(\mathbb{N})$ is the closed subspace of $l^{\infty}(\mathbb{N})$ of sequences that converge to 0 . Moreover, the function $\mathfrak{Q} f(z)$ is a bounded analytic function on $\mathbb{D}^{\infty} \cap c_{0}(\mathbb{N})$. Indeed, by the Schwarz lemma, we have, for $n<m$,

$$
\left|\mathfrak{Q} f\left(z^{(m)}\right)-\mathfrak{Q} f\left(z^{(n)}\right)\right| \leq 2\|\mathfrak{Q} f\|_{H^{\infty}\left(\mathbb{D}^{\infty}\right)} \max \left\{\left|z_{j}\right|: n<j \leq m\right\} .
$$

For $z \in \mathbb{D}^{\infty} \cap c_{0}(\mathbb{N})$, the maximum on the right hand side tends to zero as both $n$ and $m$ tend to infinity, so that the above estimate says that the $\mathfrak{Q} f\left(z^{(m)}\right)$ form a Cauchy sequence in the space of bounded analytic functions on $\mathbb{D}^{\infty} \cap c_{0}(\mathbb{N})$. We note that the supremum of $|\mathfrak{Q} f(z)|$ on $\mathbb{D}^{\infty} \cap c_{0}(\mathbb{N})$ agrees with the norm of $f$ in $H^{\infty}\left(\mathbb{D}^{\infty}\right)$.

It follows from our proof of the multiplier theorem in the next section that $H^{\infty}\left(\mathbb{D}^{\infty}\right)$ corresponds to the set of Dirichlet series representing bounded analytic functions in $\Re s>0$; in fact

$$
\|f\|_{\infty}=\|\mathfrak{Q} f\|_{H^{\infty}(\mathbb{D} \infty)} .
$$

This norm identity reflects in a concise way Bohr's observation that the $p^{-s}$ behave as "independent variables".

Thus far, we have thought about the space $H^{2}\left(\mathbb{D}^{\infty}\right)$ as the Hardy space $H^{2}$ on the infinite-dimensional polydisk $\mathbb{D}^{\infty}$. A perhaps more natural setting is to regard the spaces $H^{2}\left(\mathbb{D}^{\infty}\right)$ and $H^{\infty}\left(\mathbb{D}^{\infty}\right)$ as function spaces on the distinguished boundary $\mathbb{T}^{\infty}$. To this end, we need a group-theoretical identification of $\mathbb{T}^{\infty}$, which we shall now describe. Suppose that $\chi: \mathbb{N} \rightarrow \mathbb{C}$ satisfies

(i) $\chi(m n)=\chi(m) \chi(n), \quad m, n=1,2,3, \ldots$

(ii) $|\chi(n)|=1$.

We say that $\chi$ is a character, and write $\chi \in \Xi$. We tacitly understand that $\chi\left(n^{-1}\right)=$ $\chi(n)^{-1}$, so that the multiplicative formula holds for all positive rational numbers $\mathbb{Q}_{+}$. The characters $\chi$ constitute the dual group of $\left\langle\mathbb{Q}_{+}, \cdot\right\rangle\left(\mathbb{Q}_{+}\right.$is given the discrete topology, so that the space $\Xi$ of characters is compact). Examples of characters are the unit character $\chi(n) \equiv 1$, and, more generally, for $t \in \mathbb{R}$, 
The space $\Xi$ can be identified with the infinite-dimensional polycircle $\mathbb{T}^{\infty}$ in the following way. Given a point $z=\left(z_{1}, z_{2}, \ldots\right) \in \mathbb{T}^{\infty}$, we define the value of $\chi$ at the primes through

$$
\chi(2)=z_{1}, \quad \chi(3)=z_{2}, \ldots, \quad \chi\left(p_{m}\right)=z_{m}, \ldots,
$$

and extend the definition multiplicatively. This then yields a character, and clearly, all characters are obtained by this procedure. This provides a natural identification of the character space $\Xi$ with $\mathbb{T}^{\infty}$. The product topology on $\mathbb{T}^{\infty}$ makes it a compact space, and it is easily shown that it corresponds to the topology $\Xi$ gets as a dual group, so that the identification $\Xi=\mathbb{T}^{\infty}$ is topological. There exists a unique (apart from normalization) Haar measure on $\Xi$, which we identify with the ordinary product measure $\rho$ on $\mathbb{T}^{\infty}$. More precisely, let $\lambda$ be the arc length measure on $\mathbb{T}$, normalized so that $\lambda(\mathbb{T})=1$, and put, for Borel subsets $E_{1}, E_{2}, \ldots, E_{N}$ of $\mathbb{T}$, and $E=E_{1} \times E_{2} \times \ldots \times E_{N} \times \mathbb{T} \times \mathbb{T} \times \ldots \subset \mathbb{T}^{\infty}$,

$$
\rho(E)=\lambda\left(E_{1}\right) \lambda\left(E_{2}\right) \cdot \ldots \cdot \lambda\left(E_{N}\right)
$$

this defines a Borel measure $\rho$ on $\mathbb{T}^{\infty}$, which coincides with the Haar measure on $\Xi$, once it is agreed that the Haar measure should have total mass 1 . We shall think of $\rho$ as living on $\Xi$ as well as on $\mathbb{T}^{\infty}$.

We return to the setting of the function $f \in \mathcal{H}$ given by $(2-1)$. The series

$$
\sum_{n=1}^{\infty} a_{n} \chi(n)
$$

converges in the norm of $L^{2}(\Xi, \rho)$ (though we do not know if we have pointwise convergence almost everywhere) to a function $\mathfrak{P} f(\chi)$, which is determined uniquely by the two requirements that it be in $L^{2}(\Xi, \rho)$ and that

$$
\int_{\Xi} \bar{\chi}(q) \mathfrak{P} f(\chi) d \rho(\chi)=a_{q}, \quad q \in \mathbb{Q}_{+},
$$

where we declare $a_{q}=0$ for $q \in \mathbb{Q}_{+} \backslash \mathbb{N}$. This function $\mathfrak{P} f$ is an extension to the characters of the earlier defined function $\mathfrak{Q} f$ on the quasi-characters. Our shorthand for the above relationship between the function $\mathfrak{P} f(\chi)$ and the coefficients $\left\{a_{n}\right\}_{n}$ will be

$$
\mathfrak{P} f(\chi) \sim \sum_{n} a_{n} \chi(n), \quad \chi \in \Xi .
$$

It is clear that the Plancherel identity

$$
\int_{\Xi}|\mathfrak{P} f|^{2} d \rho=\sum_{n=1}^{\infty}\left|a_{n}\right|^{2}=\|f\|_{\mathcal{H}}^{2}
$$

holds.

Let $H^{2}(\Xi)$ (also written as $H^{2}\left(\mathbb{T}^{\infty}\right)$ ) be the closed subspace of $L^{2}(\Xi, \rho)$ consisting of functions that can be expanded in a series (2-7), with $\left\{a_{n}\right\}_{n} \in l^{2}(\mathbb{N})$. Furthermore, let $H^{\infty}(\Xi)$ be the intersection $L^{\infty}(\Xi, \rho) \cap H^{2}(\Xi)$, which is a closed subspace of $L^{\infty}(\Xi, \rho)$. To get the connection between the Hardy spaces on the distinguished boundary $\mathbb{T}^{\infty}$ and on the interior $\mathbb{D}^{\infty}$, we need the operator $\mathfrak{E}$ : $H^{2}\left(\mathbb{T}^{\infty}\right) \rightarrow H^{2}\left(\mathbb{D}^{\infty}\right)$, which associates with a function $g(\chi) \sim \sum_{n} b_{n} \chi(n)$ in $H^{2}(\Xi)$ the function $\mathfrak{E} g(\phi)=\sum_{n} b_{n} \phi(n)$ for quasi-characters $\phi \in \mathbb{D}^{\infty} \cap l^{2}(\mathbb{N})$. It is clear that it is an isometric isomorphism. The operators $\mathfrak{Q}, \mathfrak{P}$, and $\mathfrak{E}$ are related via 
Lemma 2.3. The operators $\mathfrak{P}: \mathcal{H} \rightarrow H^{2}(\Xi)$ and $\mathfrak{E}: H^{2}(\Xi) \rightarrow H^{2}\left(\mathbb{D}^{\infty}\right)$ are isometric isomorphisms. Furthermore, the restriction of $\mathfrak{E}$ to $H^{\infty}(\Xi)$ is an isometric isomorphism $H^{\infty}(\Xi) \rightarrow H^{\infty}\left(\mathbb{D}^{\infty}\right)$.

Proof. The first part just restates what was already done above. For the rest, we can refer to $[\mathbf{8}]$, where it is shown that the space $H^{\infty}(\rho)$ is canonically isometrically isomorphic to both $H^{\infty}$ on $\mathbb{D}^{\infty} \cap c_{0}(\mathbb{N})$ and on $\mathbb{D}^{\infty} \cap l^{2}(\mathbb{N})$. In $[8], H^{\infty}(\rho)$ is defined as the weak-star closure in $L^{\infty}\left(\mathbb{T}^{\infty}, \rho\right)$ of the infinite-dimensional polydisk algebra $A\left(\mathbb{D}^{\infty}\right)$. We have the inclusion $H^{\infty}(\rho) \subset H^{2}\left(\mathbb{T}^{\infty}\right)$, and by inspection, the above canonical mapping coincides with our $\mathfrak{E}$. It follows that it suffices to check that the spaces $H^{\infty}(\rho)$ and $H^{\infty}\left(\mathbb{T}^{\infty}\right)$ are the same as (closed) subspaces of $L^{\infty}(\Xi, \rho)$. Since both $H^{\infty}(\rho)$ and $H^{\infty}\left(\mathbb{T}^{\infty}\right)$ are subspaces of $H^{2}\left(\mathbb{T}^{\infty}\right)$, it is enough to check that their images under $\mathfrak{E}$ coincide. In $[8]$, it was shown that the operator $\mathfrak{E}$ was in fact furnished by integrating against the Poisson kernel in each variable, so that $\mathfrak{E}$ applied to $H^{\infty}\left(\mathbb{T}^{\infty}\right)$ consists of bounded analytic functions on $\mathbb{D}^{\infty} \cap l^{2}(\mathbb{N})$. By the above-mentioned result from [8], the assertion of the lemma follows.

2.3. Vertical limit functions. The notion of characters allows us to clarify an important property of $\mathcal{H}$. A natural unitary operator on $\mathcal{H}$ is that of vertical translation,

$$
T_{\tau} f(s)=f(s+i \tau)
$$

Fix an $f \in \mathcal{H}$. To every sequence $\tau_{n}$ of translations there exists a subsequence, say $\tau_{n(k)}$, such that $T_{\tau_{n(k)}} f(s)$ converges uniformly on compact subsets of the domain $\Re s>\frac{1}{2}$ to a limit function, say $\tilde{f}(s)$. We will say that $\tilde{f}$ is a vertical limit function of $f$. We have the following result.

Lemma 2.4. The vertical limit functions of the function $f \in \mathcal{H}$ given by (2-1) coincide with the functions of the form

$$
f_{\chi}(s)=\sum_{n=1}^{\infty} a_{n} \chi(n) n^{-s}
$$

$\chi$ being a character.

Proof. It is clear that every vertical limit function is of the form (2-8). The fact that every function of this form is a vertical limit function is a consequence of Kronecker's theorem (Lemma 2.2).

\section{The THEOREM ON MULTIPLIERS}

We put

$$
\mathbb{C}_{+}=\{s=\sigma+i t \in \mathbb{C}: \sigma>0\},
$$

and let, as usual, $H^{\infty}\left(\mathbb{C}_{+}\right)$denote the set of bounded analytic functions on $\mathbb{C}_{+}$. By $\mathcal{D}$ we denote the collection of functions $f$ which can be represented by a convergent Dirichlet series

$$
f(s)=\sum_{n=1}^{\infty} a_{n} n^{-s}
$$

in some half-plane $\sigma_{c}<\Re s<+\infty$. A multiplier $m$ on $\mathcal{H}$ is by definition a holomor- 
$f \in \mathcal{H}$. By standard functional analysis, the operation of multiplying by a multiplier is a bounded operator on $\mathcal{H}$, and the multipliers form a commutative Banach algebra. The collection of all multipliers on $\mathcal{H}$ is denoted by $\mathcal{M}$. We find it convenient to consider a multiplier both as a function and as a bounded linear operator on $\mathcal{H}$. The operator norm of $m$ is denoted by $\|m\|_{\mathcal{M}}$ and the supremum norm in $\mathbb{C}_{+}$by $\|m\|_{\infty}$.

Our theorem on multipliers can be stated as follows.

Theorem 3.1. We have $\mathcal{M}=\mathcal{D} \cap H^{\infty}\left(\mathbb{C}_{+}\right)$. Moreover, $\|m\|_{\mathcal{M}}=\|m\|_{\infty}$ holds for $m \in \mathcal{M}$.

The proof of Theorem 3.1 splits into two parts.

3.1. Proof that $\mathcal{M} \subset \mathcal{D} \cap H^{\infty}\left(\mathbb{C}_{+}\right)$and $\|m\|_{\infty} \leq\|m\|_{\mathcal{M}}$. Let $m \in \mathcal{M}$ be given. The idea of the proof is to show that $m$ lifts to a multiplier on $H^{2}\left(\mathbb{D}^{\infty}\right)$, from which we deduce that it belongs to $H^{\infty}\left(\mathbb{D}^{\infty}\right)$. This then entails that $m$ is a Dirichlet series, and that it is bounded throughout $\mathbb{C}_{+}$. Since $1 \in \mathcal{H}$, we have $m \in \mathcal{H}$, and hence

$$
m(s)=\sum_{n=1}^{\infty} b_{n} n^{-s}, \quad \Re s>\frac{1}{2},
$$

where the sequence $\left\{b_{n}\right\}_{n}$ is in $l^{2}(\mathbb{N})$. So, if we apply the operator $\mathfrak{P}$ to $m$ and $f$, where $f \in \mathcal{H}$, we get that both $\mathfrak{P} m$ and $\mathfrak{P} f$ are in $H^{2}(\Xi)$. Consequently, their product $\mathfrak{P} m \mathfrak{P} f$ is in $L^{1}(\Xi, \rho)$. Since $m$ is a multiplier, we have $m f \in \mathcal{H}$, so that $\mathfrak{P}(m f) \in H^{2}(\Xi)$. We wish to prove that $\mathfrak{P}(m f)=\mathfrak{P} m \mathfrak{P} f$ as functions in $L^{1}(\Xi, \rho)$. To this end, note that for finite Dirichlet series $f$, this is verified by direct calculation. The general case when $f \in \mathcal{H}$ is arbitrary then follows by approximating $f$ with finite Dirichlet series.

Since $\|m f\|_{\mathcal{H}} \leq\|m\|_{\mathcal{M}}\|f\|_{\mathcal{H}}$, we get, by successively plugging in $f=1, m, m^{2}$, and so on, that $\left\|m^{j}\right\|_{\mathcal{H}} \leq\|m\|_{\mathcal{M}}^{j}$ holds for $j=1,2, \ldots$ By what we just did, $\mathfrak{P}\left(m^{j}\right)=(\mathfrak{P} m)^{j}$, so that

$$
\left(\int_{\Xi}|\mathfrak{P} m|^{2 j} d \rho\right)^{1 /(2 j)}=\left\|(\mathfrak{P} m)^{j}\right\|_{H^{2}(\Xi)}^{1 / j}=\left\|m^{j}\right\|_{\mathcal{H}}^{1 / j} \leq\|m\|_{\mathcal{M}} .
$$

As $j \rightarrow+\infty$, the left hand side tends to $\|\mathfrak{P} m\|_{L^{\infty}(\Xi, \rho)}$. We conclude that $\mathfrak{P} m$ belongs to $L^{\infty}(\Xi, \rho)$ as well as $H^{2}(\Xi)$, that is, $\mathfrak{P} m \in H^{\infty}(\Xi)$. It also follows that $\|\mathfrak{P} m\|_{H^{\infty}(\Xi)} \leq\|m\|_{\mathcal{M}}$. By Lemma 2.3, the function $\mathfrak{Q} m=\mathfrak{E P} m$ is in $H^{\infty}\left(\mathbb{D}^{\infty}\right)$, and its norm equals the norm of $\mathfrak{P} m$ in $H^{\infty}(\Xi)$. Recall that by Hilbert's approach to power series of infinitely many variables, the functions in the space $H^{\infty}\left(\mathbb{D}^{\infty}\right)$ are bounded and analytic in $\mathbb{D}^{\infty} \cap c_{0}(\mathbb{N})$, and that the formula giving $\mathfrak{Q} m$ on (part of) the quasi-characters is

$$
\mathfrak{Q} m(\phi)=\sum_{n=1}^{\infty} b_{n} \phi(n), \quad \phi \in \mathbb{D}^{\infty} \cap l^{2}(\mathbb{N}) .
$$

Plugging in the special quasi-character $\phi_{s}(n)=n^{-s}$, we get our function $m(s)$ back: $m(s)=\mathfrak{Q} m\left(\phi_{s}\right)$ for $\Re s>\frac{1}{2}$. Since $\phi_{s}$ depends analytically on the parameter $s$ and is in $\mathbb{D}^{\infty} \cap c_{0}(\mathbb{N})$ for $\Re s>0$, the fact that $\mathfrak{Q} m$ is bounded and holomorphic on $\mathbb{D}^{\infty} \cap c_{0}(\mathbb{N})$ implies that $m(s)$ is bounded and analytic in $\mathbb{C}_{+}$, and $\|m\|_{\infty} \leq$ 
3.2. Proof that $\mathcal{D} \cap H^{\infty}\left(\mathbb{C}_{+}\right) \subset \mathcal{M}$ and $\|m\|_{\mathcal{M}} \leq\|m\|_{\infty}$. The key to the proof of the converse relation is the following lemma, which is due to Fritz Carlson [7].

Lemma 3.2. (Carlson's theorem) Let $f(s)=\sum_{n=1}^{\infty} a_{n} n^{-s}$ be convergent (and hence analytic) in $\mathbb{C}_{+}$and bounded in every half-plane $\Re(s)>\delta$ with $\delta>0$. Then, for each $\sigma>0$,

$$
\sum_{n=1}^{\infty}\left|a_{n}\right|^{2} n^{-2 \sigma}=\lim _{T \rightarrow+\infty} \frac{1}{2 T} \int_{-T}^{T}|f(\sigma+i t)|^{2} d t .
$$

An immediate corollary is the following.

Lemma 3.3. If $f(s)=\sum_{n=1}^{\infty} a_{n} n^{-s}$ is convergent and bounded in $\mathbb{C}_{+}$, then $f \in \mathcal{H}$ and

$$
\|f\|_{\mathcal{H}}=\lim _{\sigma \rightarrow 0^{+}}\left(\lim _{T \rightarrow+\infty} \frac{1}{2 T} \int_{-T}^{T}|f(\sigma+i t)|^{2} d t\right)^{1 / 2} .
$$

Remark. Note that together with Lemma 2.1, Lemma 3.3 implies Bohr's inequality $\sigma_{a}-\sigma_{u} \leq \frac{1}{2}$.

We carry on with the proof, and suppose that

$$
m(s)=\sum_{n=1}^{\infty} b_{n} n^{-s}
$$

converges in some half-plane $\Re s>\sigma_{0}$, and that it extends boundedly and holomorphically to $\mathbb{C}_{+}$. Then, by Bohr's theorem (Lemma 2.1), the Dirichlet series defining $m(s)$ actually converges uniformly to $m(s)$ on every half-plane $\Re s>\varepsilon$, with $\varepsilon>0$. Let $f \in \mathcal{H}$ have Dirichlet series $\sum_{n=1}^{\infty} a_{n} n^{-s}$, and introduce, for $N=1,2,3, \ldots$, the cut-off series

$$
f_{N}(s)=\sum_{n=1}^{N} a_{n} n^{-s}, \quad s \in \mathbb{C} .
$$

The function $m f_{N}$ is given by a convergent Dirichlet series in $\mathbb{C}_{+}$, and it is bounded there. We can now apply Lemma 3.3 to the function $m f_{N}$, to obtain

$$
\left\|m f_{N}\right\|_{\mathcal{H}} \leq\|m\|_{\infty}\left\|f_{N}\right\|_{\mathcal{H}} \leq\|m\|_{\infty}\|f\|_{\mathcal{H}} .
$$

Since $\|m f\|_{\mathcal{H}} \leq \sup _{N}\left\|m f_{N}\right\|_{\mathcal{H}}$, it follows that

$$
\|m f\|_{\mathcal{H}} \leq\|m\|_{\infty}\|f\|_{\mathcal{H}},
$$

which completes the proof of Theorem 3.1.

Remark. In the proof of Theorem 3.1, we actually prove that the multipliers on $\mathcal{H}$ may be identified with the space $H^{\infty}\left(\mathbb{D}^{\infty}\right)$. It is remarkable that just being able to extend the function $m \in \mathcal{H}$ holomorphically and boundedly to $\mathbb{C}_{+}$should entail that the function $\mathfrak{Q} m \in H^{2}\left(\mathbb{D}^{\infty}\right)$ is bounded on $\mathbb{D}^{\infty} \cap l^{2}(\mathbb{N})$ (and hence bounded and analytic on $\mathbb{D}^{\infty} \cap c_{0}(\mathbb{N})$ ). After all, the condition on $m$ just corresponds to the behavior of $\mathfrak{Q} m$ along the one-dimensional complex variety which is the image of 


\section{Some function theoretic properties of $\mathcal{H}$}

We first recall some results from ergodic theory. We then turn to the almost sure behavior of the vertical limit functions $f_{\chi}$, with $\chi \in \Xi$, of a given $f \in \mathcal{H}$. As an application, we consider the famous zeta function, which here plays the role of the kernel function. After that, we look at the function-theoretic properties of individual functions in $\mathcal{H}$; in particular, we study zero sets.

4.1. Preliminaries from ergodic theory: Kronecker flows. Given a collection of real numbers $\alpha_{1}, \alpha_{2}, \alpha_{3}, \ldots$, we consider the continuous group of vertical translations

$$
T_{t}\left(z_{1}, z_{2}, z_{3}, \ldots\right)=\left(e^{-i t \alpha_{1}} z_{1}, e^{-i t \alpha_{2}} z_{2}, e^{-i t \alpha_{3}} z_{3}, \ldots\right),
$$

acting on the infinite-dimensional polycircle $\mathbb{T}^{\infty}$, where $t$ ranges over the reals. The Kronecker flow $\left\{T_{t}\right\}_{t}$ is known to be ergodic if and only if for each fixed $n, n=1,2,3, \ldots$, the numbers $\alpha_{1}, \alpha_{2}, \ldots, \alpha_{n}$ are $\mathbb{Q}$-linearly independent. This is done for finite-dimensional polycircles $\mathbb{T}^{n}$ in $[\mathbf{9}$, pp. 64, 67, 69, 99]. After a few minor modifications, the proof in [9], which is based on the approximation property in Kronecker's theorem (Lemma 2.2), covers the infinite-dimensional case as well. We pick $\alpha_{j}=\log p_{j}$, where $p_{j}$ denotes the $j$-th prime, and note that by the fundamental theorem of arithmetic, any finite subset of the collection $\left\{\alpha_{j}\right\}_{j}$ is $\mathbb{Q}$-linearly independent, so that the flow $\left\{T_{t}\right\}_{t}$ is ergodic. If we write out the flow explicitly, we get

$$
T_{t}\left(z_{1}, z_{2}, z_{3}, \ldots\right)=\left(2^{-i t} z_{1}, 3^{-i t} z_{2}, 5^{-i t} z_{3}, \ldots\right), \quad t \in \mathbb{R} .
$$

As in Section 2 , points $z=\left(z_{1}, z_{2}, \ldots\right)$ in $\mathbb{T}^{\infty}$ are identified with elements $\chi$ of the character group $\Xi$ by putting $\chi\left(p_{j}\right)=z_{j}$ for the $j$-th prime $p_{j}$. The flow then takes the more elegant form

$$
\left(T_{t} \chi\right)(n)=n^{-i t} \chi(n), \quad n \in \mathbb{N}, t \in \mathbb{R} .
$$

We note that the ergodicity of the flow $\left\{T_{t}\right\}_{t}$ may now be checked off directly from condition (iv) in $[\mathbf{9}$, p. 99]. By the Birkhoff-Khinchin ergodic theorem [9, pp. 11-12, 39, 99], we have

$$
\lim _{T \rightarrow+\infty} \frac{1}{2 T} \int_{-T}^{T} g\left(T_{t} \chi_{0}\right) d t=\int_{\Xi} g(\chi) d \rho(\chi)
$$

for every $\chi_{0} \in \Xi$ if $g$ is continuous on $\Xi$, and for almost every $\chi_{0}$ if we only assume that $g \in L^{1}(\Xi, \rho)$. We now apply this result in the context of our space $\mathcal{H}$. For $f \in \mathcal{H}$, with series expansion $f(s)=\sum_{n} a_{n} n^{-s}$, we write $f_{\sigma}(s)=f(\sigma+s)=$ $\sum_{n} a_{n} n^{-\sigma-s}$, and note that for $\sigma \geq 0$, this is again an element of $\mathcal{H}$. Recall that $\sigma_{b}(f) \in\left[-\infty, \frac{1}{2}\right]$ is the abscissa of boundedness for $f$, and that by Bohr's theorem (Lemma 2.1), it coincides with the abscissa of uniform convergence. It follows that for $\sigma, \sigma_{b}(f)<\sigma<+\infty$, the Dirichlet series for $f_{\sigma}(s)$ is uniformly convergent on $\Re s>0$, so that by Kronecker's theorem (Lemma 2.2), the partial sums of the series $\mathfrak{P} f_{\sigma}$ converge uniformly on $\Xi$, making $\mathfrak{P} f_{\sigma}$ continuous on $\Xi$. Now, by $(4-1)$,

$$
\lim _{T \rightarrow+\infty} \frac{1}{2 T} \int_{-T}^{T}\left|\mathfrak{P} f_{\sigma}\left(T_{t} \chi_{0}\right)\right|^{2} d t=\int_{\Xi}\left|\mathfrak{P} f_{\sigma}(\chi)\right|^{2} d \rho(\chi)=\sum_{n=1}^{\infty}\left|a_{n}\right|^{2} n^{-2 \sigma}
$$

holds for almost all $\chi_{0} \in \Xi$ if $0 \leq \sigma \leq \sigma_{b}(f)$, and for all $\chi_{0}$ (in particular for the unit character $\chi_{0} \equiv 1$ ) if $\sigma>\frac{1}{2}$. Notice the close resemblance with Carlson's theorem (Lemma 3.2). Indeed, Carlson's theorem can be read off from (4-2), with 
4.2. The almost sure behavior of vertical limit functions. As before, let $f \in \mathcal{H}$ be given by (2-1), and write $f_{\chi}$ for the vertical limit function of $f$, given by $(2-8)$. The function $f_{\chi}$ shares with $f$ the property that it is holomorphic on $\Re s>\frac{1}{2}$. Helson [16] has shown that the function $f_{\chi}$ extends analytically to $\Re s>0$ and that its Dirichlet series converges there, for almost every $\chi \in \Xi$. We wish to illuminate his argument, and obtain additional properties of the vertical limit functions. To make the statement as precise as possible, we need the space $H_{\mathrm{i}}^{2}\left(\mathbb{C}_{+}\right)$of functions $f$ holomorphic in $\mathbb{C}_{+}$which have $f \circ \varphi \in H^{2}(\mathbb{D})$; here $H^{2}(\mathbb{D})$ is the usual Hardy space of the unit disk $\mathbb{D}$, and $\varphi$ is the Cayley transform $\varphi(z)=(1-z) /(1+z)$. The subscript "i" stands for (conformal) invariance. Let $\lambda_{\mathrm{i}}$ be the probability measure $d \lambda_{\mathrm{i}}(t)=\pi^{-1}\left(1+t^{2}\right)^{-1}$, which is obtained as the image under the Cayley transform of the normalized arc length measure on the unit circle:

$$
\int_{-\infty}^{+\infty}|f(i t)|^{2} d \lambda_{\mathrm{i}}(t)=\frac{1}{2 \pi} \int_{-\pi}^{\pi}\left|f \circ \varphi\left(e^{i \theta}\right)\right|^{2} d \theta, \quad f \in H_{\mathrm{i}}^{2}\left(\mathbb{C}_{+}\right)
$$

The space $H^{2}(\mathbb{D})$ is usually regarded as a subspace of $L^{2}(\mathbb{T})$, and likewise the space $H_{\mathrm{i}}^{2}\left(\mathbb{C}_{+}\right)$may be considered to be a subspace of $L^{2}\left(i \mathbb{R}, \lambda_{\mathrm{i}}^{*}\right)$, the space of functions $g$ on $i \mathbb{R}$ that have $g(i t)$ in $L^{2}\left(\mathbb{R}, \lambda_{\mathrm{i}}\right)$.

Apart from the ergodic statement, the following theorem is due to Helson [14]. For the benefit of the reader, we adapt Helson's proof to the present setting.

Theorem 4.1. Let $f \in \mathcal{H}$ be given, with series expansion (2-1), and let $\Pi$ be a countable collection of absolutely continuous Borel probability measures on the real line. For almost every character $\chi \in \Xi$, the function

$$
f_{\chi}(s)=\sum_{n=1}^{\infty} a_{n} \chi(n) n^{-s}, \quad \Re s>\frac{1}{2}
$$

extends analytically to an element of $H_{\mathrm{i}}^{2}\left(\mathbb{C}_{+}\right)$, has

$$
\int_{-\infty}^{+\infty}\left|f_{\chi}(i t)\right|^{2} d \varpi(t)<+\infty, \quad \text { for all } \varpi \in \Pi
$$

and enjoys

$$
\frac{1}{2 T} \int_{-T}^{T}\left|f_{\chi}(i t)\right|^{2} d t \rightarrow \sum_{n=1}^{\infty}\left|a_{n}\right|^{2} \quad \text { as } \quad T \rightarrow+\infty
$$

Proof. We first decide on how to define $f_{\chi}(i t)$. It should correspond to the possibly divergent sum $\sum_{n} a_{n} \chi(n) n^{-i t}$. This sum, however, makes sense as $\mathfrak{P} f\left(T_{t} \chi\right)$, for almost every $\chi$, where $\left\{T_{t}\right\}_{t}$ is the ergodic Kronecker flow of the previous subsection. We thus put $f_{\chi}(i t)=\mathfrak{P} f\left(T_{t} \chi\right)$, and observe that by (4-2), with $\sigma=0$, this function is locally in $L^{2}$ along the imaginary axis, and has the asserted property

$$
\frac{1}{2 T} \int^{T}\left|f_{\chi}(i t)\right|^{2} d t \rightarrow \sum^{\infty}\left|a_{n}\right|^{2} \quad \text { as } \quad T \rightarrow+\infty
$$


almost surely in $\chi$. Moreover, by Fubini's theorem, for a Borel probability measure $\varpi$

$$
\begin{aligned}
\int_{\Xi} \int_{-\infty}^{+\infty}\left|f_{\chi}(i t)\right|^{2} d \varpi(t) d \rho(\chi)=\int_{-\infty}^{+\infty} \int_{\Xi}\left|f_{\chi}(i t)\right|^{2} d \rho(\chi) d \varpi(t) & \\
& =\int_{-\infty}^{+\infty}\|f\|_{\mathcal{H}}^{2} d \varpi(t)=\|f\|_{\mathcal{H}}^{2}<+\infty .
\end{aligned}
$$

In particular, the function $f_{\chi}(i t)$, considered as a function of $t$, almost surely is square integrable on the real line with respect to $\varpi$. Elementary measure theory shows that the same holds true simultaneously for all measures $\varpi$ in $\Pi$, since the latter set is countable. By the same token, $f_{\chi}(i t)$ is almost surely in $L^{2}\left(\mathbb{R}, \lambda_{\mathrm{i}}\right)$.

A function $g$ in $L^{2}(\mathbb{T})$ is in $H^{2}(\mathbb{D})$ if and only if

$$
\int_{\mathbb{T}} z^{n} g(z) d \lambda(z)=0, \quad n=1,2,3, \ldots
$$

where $\lambda$ is as before the normalized arc length measure on the unit circle. After an application of the Cayley transform, we have that a function $g \in L^{2}\left(i \mathbb{R}, \lambda_{\mathrm{i}}^{*}\right)$ is in $H_{\mathrm{i}}^{2}\left(\mathbb{C}_{+}\right)$if and only if

$$
\int_{-\infty}^{+\infty}\left(\frac{1-i t}{1+i t}\right)^{n} g(i t) d \lambda_{\mathrm{i}}(t)=0, \quad n=1,2,3, \ldots
$$

Therefore, to see that $f_{\chi}$ is almost surely in $H_{\mathrm{i}}^{2}\left(\mathbb{C}_{+}\right)$, it suffices to check that

$$
\int_{-\infty}^{+\infty}\left(\frac{1-i t}{1+i t}\right)^{n} f_{\chi}(i t) d \lambda_{\mathrm{i}}(t)=0, \quad n=1,2,3, \ldots
$$

for almost all $\chi$. However, to check that an $L^{2}(\Xi, \rho)$ function vanishes almost everywhere, it is enough to show that all of its Fourier coefficients are 0. We set $a_{q}=0$ for $q \in \mathbb{Q}_{+} \backslash \mathbb{N}$, and integrate the left hand side of the above expression against $\bar{\chi}(q)$ to get the Fourier coefficients:

$$
\begin{aligned}
& \int_{\Xi} \bar{\chi}(q) \int_{-\infty}^{+\infty}\left(\frac{1-i t}{1+i t}\right)^{n} f_{\chi}(i t) d \lambda_{\mathrm{i}}(t) \\
= & \int_{-\infty}^{+\infty}\left(\frac{1-i t}{1+i t}\right)^{n} \int_{\Xi} \bar{\chi}(q) f_{\chi}(i t) d \rho(\chi) d \lambda_{\mathrm{i}}(t)=\int_{-\infty}^{+\infty}\left(\frac{1-i t}{1+i t}\right)^{n} a_{q} q^{-i t} d \lambda_{\mathrm{i}}(t)=0,
\end{aligned}
$$

where we have used Fubini's theorem, and that $a_{q}=0$ for $q<1$. This completes the proof.

The ergodic reasoning behind (4-4) leads to an estimate of $f_{\chi}$, which does not seem to follow from Helson's work.

Theorem 4.2. Let $f$ and $f_{\chi}$ be as in Theorem 4.1, and write $s=\sigma+i t$. Then almost surely in $\chi \in \Xi$,

$$
\left|f_{\chi}(s)-a_{1}\right| \leq C\left(\frac{1+|t|^{1 / 2}}{1^{/ 0}}\right), \quad s \in \mathbb{C}_{+},
$$


for some constant $C=C(f, \chi), 0<C<+\infty$. Moreover, almost surely in $\chi$,

$$
f_{\chi}(s)=a_{1}+o\left(\frac{|t|^{1 / 2}}{\sigma^{1 / 2}}\right) \quad \text { as } \quad|t| \rightarrow+\infty
$$

holds uniformly in $\sigma>0$.

Proof. As in (4-4), we have, by ergodic theory, that almost surely in $\chi$,

$$
\frac{1}{T} \int_{0}^{T}\left(f_{\chi}(i \tau)-a_{1}\right)^{2} d \tau \rightarrow 0 \quad \text { as } \quad|T| \rightarrow+\infty,
$$

the space average of the function (in $\chi$ ) being 0 . This entails that the function

$$
F_{\chi}(i x)=\int_{0}^{x}\left(f_{\chi}(i \tau)-a_{1}\right)^{2} d \tau
$$

meets

$$
F_{\chi}(i x)=o(|x|), \quad \text { as } \quad|x| \rightarrow+\infty,
$$

almost surely in $\chi$. By Theorem 4.1, the function $f_{\chi}$ is almost surely in $H_{\mathrm{i}}^{2}\left(\mathbb{C}_{+}\right)$, so that the squared function $\left(f_{\chi}-a_{1}\right)^{2}$ almost surely belongs to the analogously defined space $H_{\mathrm{i}}^{1}\left(\mathbb{C}_{+}\right)$. As such it is given by the Poisson formula

$$
\left(f_{\chi}(\sigma+i t)-a_{1}\right)^{2}=\frac{\sigma}{\pi} \int_{-\infty}^{+\infty} \frac{\left(f_{\chi}(i x)-a_{1}\right)^{2}}{\sigma^{2}+(t-x)^{2}} d x .
$$

Integrating by parts, using (4-5), we obtain the representation formula

$$
\left(f_{\chi}(\sigma+i t)-a_{1}\right)^{2}=\frac{2 \sigma}{\pi} \int_{-\infty}^{+\infty} \frac{t-x}{\left(\sigma^{2}+(t-x)^{2}\right)^{2}} F_{\chi}(i x) d x
$$

for $\sigma>0$ and $t \in \mathbb{R}$. After an application of the size control (4-5) to this integral, the desired estimates follow by taking square roots.

For a certain class of functions in $\mathcal{H}$, the estimate of Theorem 4.2 can be improved considerably. Note that the conclusion is that the growth in the imaginary direction is precisely what the Schnee-Landau theorem requires to imply convergence of Dirichlet series $[\mathbf{5}, \mathbf{2 0}]$.

Corollary 4.3. Let $f \in \mathcal{H}$ be such that all powers $f^{N}$, where $N$ is a positive integer, also are in $\mathcal{H}$. Then, for each $\varepsilon>0$, we have, almost surely in $\chi \in \Xi$,

$$
\left|f_{\chi}(s)-a_{1}\right| \leq C\left(\frac{1+|t|^{\varepsilon}}{\sigma^{\varepsilon}}\right), \quad s=\sigma+i t \in \mathbb{C}_{+},
$$

for some constant $C=C(\chi, f, \varepsilon), 0<C<+\infty$.

Proof. The estimate is more or less immediate from Theorem 4.2.

The general question about almost sure convergence of the Dirichlet series of vertical limit functions was treated by Helson in [16], in a somewhat more general 
Theorem 4.4. (Helson) Let $f \in \mathcal{H}$ be given, with series expansion (2-1). For almost every character $\chi$, the Dirichlet series

$$
f_{\chi}(s)=\sum_{n} a_{n} \chi(n) n^{-s}
$$

converges in the half-plane $\Re s>0$.

According to a classical formula for the abscissa of convergence [12, pp. 6-8] (see also Section 2 of the present paper), Theorem 4.4 is equivalent to the statement that, almost surely in $\chi$, the partial sum function

$$
S_{N}(\chi)=\sum_{n: n \leq N} a_{n} \chi(n)
$$

has $S_{N}(\chi)=O\left(N^{\varepsilon}\right)$ as $N \rightarrow+\infty$, almost surely in $\chi$, for all $\varepsilon>0$. So, in light of Helson's theorem, we find the following special case interesting.

Theorem 4.5. Let $f \in \mathcal{H}$ be given, with series expansion (2-1). Suppose $a_{n}=0$ for all composite numbers. For almost every character $\chi$, we then have $S_{N}(\chi)=O(1)$ as $N \rightarrow \infty$, where $S_{N}(\chi)$ is as in (4-6).

Proof. The non-composite numbers are the primes and unity (1). Without loss of generality, we may suppose that $a_{1}=0$. As the parameter $p$ runs through the primes, the $\chi(p)$, treated as functions of $\chi$, run through the distinct coordinate variables in the polycircle $\mathbb{T}^{\infty}$, which is our standard realization of the character space $\Xi$. This has a clear interpretation: the random variables

$$
\chi \mapsto a_{p} \chi(p), \quad p=2,3,5,7,11, \ldots
$$

are mutually independent, have mean squares 0 , and variances $\sigma_{p}^{2}=\left|a_{p}\right|^{2}$.

For a given positive integer $N$ and a positive real number $M$, let $E(N, M)$ be the set of all characters $\chi$ for which $\left|S_{N}(\chi)\right|<M$. By Kolmogorov's inequality [9, p. 260], we have

$$
\rho\left(\cap_{N} E(N, M)\right) \geq 1-M^{-2} \sum_{p} \sigma_{p}^{2}=1-M^{-2}\left|a_{p}\right|^{2}
$$

By letting $M$ tend to $+\infty$, we see that $S_{N}(\chi)=O(1)$ as $N \rightarrow+\infty$ holds for almost all $\chi \in \Xi$.

4.3. The kernel function. Riemann's zeta function for random characters. Given a separable Hilbert space $\mathcal{A}$ of analytic functions on a domain $\Omega$ in $\mathbb{C}$ or in $\mathbb{C}^{n}$, one forms the kernel function $K_{\mathcal{A}}(z, w)$ by taking some orthonormal basis $\left\{e_{n}(z)\right\}_{n}$ in $\mathcal{A}$, and putting

$$
K_{\mathcal{A}}(z, w)=\sum_{n} e_{n}(z) \bar{e}_{n}(w), \quad(z, w) \in \Omega \times \Omega
$$

This then proves to be independent of the particular choice of orthonormal basis, and has the reproducing property that 
In fact, as an element of $\mathcal{A}, K(\cdot, w)$ is uniquely determined by its reproducing property. In $\mathcal{H}$, an orthonormal basis is supplied by $e_{n}(z)=n^{-z}$, for $n=1,2,3, \ldots$, so that its kernel function is

$$
K_{\mathcal{H}}(z, w)=\sum_{n=1}^{\infty} n^{-z-\bar{w}}=\zeta(z+\bar{w}), \quad \Re z>\frac{1}{2}, \Re w>\frac{1}{2},
$$

where $\zeta(s)$ is the Riemann zeta function:

$$
\zeta(s)=\sum_{n=1}^{\infty} n^{-s}, \quad \Re s>1 .
$$

In Section 2, we modeled the space $\mathcal{H}$ as both the Hardy space on the infinitedimensional polydisk $\mathbb{D}^{\infty}$ and the polycircle $\mathbb{T}^{\infty}$. The polycircle $\mathbb{T}^{\infty}$ was identified with the character group $\Xi$ of the multiplicative positive rationals. In particular, for quasi-characters $\phi \in \mathbb{D}^{\infty} \cap l^{2}(\mathbb{N})$, the "point evaluation" $f \mapsto \mathfrak{Q} f(\phi)$ is a continuous linear functional on $\mathcal{H}$, so that it too must be given by a kernel function $K_{H^{2}(\mathbb{D} \infty)}(\psi, \phi)$,

$$
\mathfrak{Q} f(\phi)=\left\langle\mathfrak{Q} f, K_{H^{2}(\mathbb{D} \infty)}(\cdot, \phi)\right\rangle_{H^{2}(\mathbb{D} \infty)}, \quad \phi \in \mathbb{D}^{\infty} \cap l^{2}(\mathbb{N}) .
$$

In terms of a series expansion, it is written

$$
K_{H^{2}\left(\mathbb{D}^{\infty}\right)}(\psi, \phi)=\sum_{n=1}^{\infty} \psi(n) \bar{\phi}(n), \quad \psi, \phi \in \mathbb{D}^{\infty} \cap l^{2}(\mathbb{N}) .
$$

The inner product in $\mathcal{H}$, and hence in $H^{2}\left(\mathbb{D}^{\infty}\right)$, is better visualized on the distinguished boundary $\mathbb{T}^{\infty}=\Xi$, where we have

$$
\begin{aligned}
\langle f, g\rangle_{\mathcal{H}}=\langle\mathfrak{Q} f, \mathfrak{Q} g\rangle_{H^{2}\left(\mathbb{D}^{\infty}\right)}=\langle\mathfrak{P} f, \mathfrak{P} g\rangle_{H^{2}\left(\mathbb{T}^{\infty}\right)} & \\
& =\int_{\Xi} \mathfrak{P} f(\chi) \overline{\mathfrak{P} g}(\chi) d \rho(\chi), \quad f, g \in \mathcal{H} .
\end{aligned}
$$

This suggests introducing the kernel

$$
K_{H^{2}(\Xi)}(\chi, \phi)=\sum_{n=1}^{\infty} \chi(n) \bar{\phi}(n), \quad \chi \in \Xi, \phi \in \mathbb{D}^{\infty} \cap l^{2}(\mathbb{N}),
$$

where the sum, for fixed $\phi$, is understood to converge in the sense of the space $L^{2}(\Xi, \rho)$. This kernel then has the reproducing property

$$
\begin{aligned}
& \mathfrak{Q} f(\phi)=\left\langle\mathfrak{P} f, K_{H^{2}(\Xi)}(\cdot, \phi)\right\rangle_{H^{2}(\Xi)} \\
& \quad=\int_{\Xi} \mathfrak{P} f(\chi) \overline{K_{H^{2}(\Xi)}}(\chi, \phi) d \rho(\chi), \quad \phi \in \mathbb{D}^{\infty} \cap l^{2}(\mathbb{N}) .
\end{aligned}
$$

On the other hand, it is well known that the reproducing kernel on $H^{2}\left(\mathbb{T}^{N}\right)$ for finite-dimensional polydisks is given as the product of the Cauchy kernel in each variable,

$$
K_{H^{2}\left(\mathbb{T}^{N}\right)}(z, w)=\prod^{N}\left(1-\bar{w}_{n} z_{n}\right)^{-1}, \quad z \in \mathbb{T}^{N}, w \in \mathbb{D}^{N}
$$


and in the limit as the dimension $N$ tends to infinity, we get $[\mathbf{8}]$

$$
\begin{aligned}
K_{H^{2}\left(\mathbb{T}^{\infty}\right)}(\chi, \phi)=\prod_{n=1}^{\infty}(1 & \left.-\bar{w}_{n} z_{n}\right)^{-1} \\
& =\prod_{p}(1-\bar{\phi}(p) \chi(p))^{-1}, \quad \chi \in \mathbb{T}^{\infty}, \phi \in \mathbb{D}^{\infty} \cap l^{2}(\mathbb{N}),
\end{aligned}
$$

where the second product runs over the primes, and $z_{n}=\chi\left(p_{n}\right), w_{n}=\phi\left(p_{n}\right)$, for the $n$-th prime $p_{n}$. For $\phi \in \mathbb{D}^{\infty} \cap l^{1}(\mathbb{N})$, the above product converges pointwise to a continuous function of $\chi$, but in general we must interpret the above product as being convergent in $L^{2}(\Xi, \rho)[\boldsymbol{8}]$ (Cole and Gamelin used martingale theory to obtain the convergence). Since the kernel function of a given Hilbert space of analytic functions is unique, we arrive at the equality $K_{H^{2}(\Xi)}=K_{H^{2}\left(\mathbb{T}^{\infty}\right)}$, that is, the Euler identity

$$
\sum_{n=1}^{\infty} \chi(n) \bar{\phi}(n)=\prod_{p}(1-\chi(p) \bar{\phi}(p))^{-1}, \quad \chi \in \mathbb{T}^{\infty}, \phi \in \mathbb{D}^{\infty} \cap l^{2}(\mathbb{N}),
$$

which holds pointwise in $\chi$ for $\phi \in \mathbb{D}^{\infty} \cap l^{1}(\mathbb{N})$, and almost everywhere for general $\phi$. This is a surprising interpretation of the Euler identity as arising from two ways of looking at one and the same kernel function! If we specialize to the particular quasi-characters $\phi(n)=n^{-\bar{s}}$, we get the more familiar

$$
\sum_{n=1}^{\infty} \chi(n) n^{-s}=\prod_{p}\left(1-\chi(p) p^{-s}\right)^{-1}, \quad \chi \in \Xi, \Re s>\frac{1}{2},
$$

with pointwise convergence in $\chi$ for $\Re s>1$. We shall write $\zeta_{\chi}(s)$ for the analytic function on $\Re s>1$ given by either side of (4-7). By Lemma 2.4, suitably modified, these are the vertical limit functions of the zeta function $\zeta(s)$.

Theorem 4.6. Suppose the coefficients $\left\{a_{n}\right\}_{n}$ are totally multiplicative and square summable, with $a_{1}=1$. Then, for almost every character $\chi$, the Dirichlet series

$$
f_{\chi}(s)=\sum_{n=1}^{\infty} a_{n} \chi(n) n^{-s}, \quad \Re s>\frac{1}{2},
$$

converges to a zero-free analytic function on the half-plane $\Re s>0$.

Proof. The convergence statement follows from Theorem 4.4. We check that the function $f_{\chi}(s)$ almost surely lacks zeros in $\Re s>0$. Let $\mu(n)$ be the Möbius function, which has $\mu(1)=1, \mu(n)=(-1)^{k}$ if $n$ is the product of $k$ different primes, and $\mu(n)=0$ if $n$ is divisible by a square (other than 1). The Möbius function enables us to express the reciprocal of $f_{\chi}(s)$,

$$
1 / f_{\chi}(s)=\sum_{n=1}^{\infty} \mu(n) a_{n} \chi(n) n^{-s}, \quad \Re s>1,
$$

because the coefficients $\left\{a_{n}\right\}_{n}$ are totally multiplicative. By Theorem $4.1,1 / f_{\chi}(s)$ extends analytically to $\mathbb{C}_{+}$almost surely in $\chi$, whence the assertion follows.

Theorem 4.6 has a curious interpretation for the vertical limit functions $\zeta_{\chi}(s)$ of the Riemann zeta function: the Riemann hypothesis holds for almost every $\chi$ (see 
Corollary 4.7. (Helson) For almost every character $\chi$, the Dirichlet series $\zeta_{\chi}(s)=$ $\sum_{n} \chi(n) n^{-s}$ converges on the half-plane $\Re s>\frac{1}{2}$ to an analytic function which has no zeros there.

Proof. Apply Theorems 4.1 and 4.6 to the coefficients $a_{n}=n^{-\frac{1}{2}-\varepsilon}$, with $\varepsilon>0$. The assertion follows, but only in the slightly smaller half-plane $\Re s>\frac{1}{2}+\varepsilon$. An elementary measure-theoretical argument now yields the desired result.

By the formula for the abscissa of convergence of a Dirichlet series (see Section 2), Corollary 4.7 has the following consequence.

Corollary 4.8. Let $\varepsilon>0$. For almost every character $\chi$, we have $\sum_{n=1}^{N} \chi(n)=$ $O\left(N^{1 / 2+\varepsilon}\right)$ as $N \rightarrow+\infty$.

Question. Can the above be sharpened to the statement that, almost surely, $\sum_{n=1}^{N} \chi(n)=O(\sqrt{N} \log N)$ as $N \rightarrow+\infty$ ?

Using Corollary 4.3, we can control the growth of the logarithm of $\zeta_{\chi}(s)$. The branch of the logarithm intended is the one with

$$
\log \zeta_{\chi}(s)=-\sum_{p} \log \left(1-\chi(p) p^{-s}\right), \quad \Re s>1,
$$

where the logarithms on the right hand side are given by the principal branch.

Theorem 4.9. Suppose the coefficients $\left\{a_{n}\right\}_{n}$ are totally multiplicative and square summable, with $a_{1}=1$. Then, for almost every character $\chi$, the function $f_{\chi}(s)=$ $\sum_{n} a_{n} \chi(n) n^{-s}$ has a logarithm

$$
\log f_{\chi}(s)=-\sum_{p} \log \left(1-a_{p} \chi(p) p^{-s}\right), \quad \Re s>\frac{1}{2},
$$

(the right hand side involves the principal branch) which extends holomorphically to $\Re s>0$, and enjoys $(s=\sigma+i t)$

$$
\log f_{\chi}(s)=o\left(\log \frac{|t|}{\sigma}\right) \quad \text { as } \quad|t| \rightarrow+\infty,
$$

uniformly in $\sigma, \sigma>0$.

Proof. Let $\alpha$ be a complex parameter. We define the power

$$
f(s)^{\alpha}=\prod_{p}\left(1-a_{p} p^{-s}\right)^{-\alpha}, \quad \Re s>\frac{1}{2},
$$

where the right hand side employs the principal branch of the logarithm. The identification of $\mathcal{H}$ with $H^{2}(\Xi)$ in Section 2 allows us to express the square of the norm of $f^{\alpha}$ in $\mathcal{H}$ as

$$
\prod_{p} \frac{1}{2 \pi} \int_{-\pi}^{\pi}\left|\left(1-a_{p} e^{i \theta}\right)^{-\alpha}\right|^{2} d \theta
$$

Using a Maclaurin expansion of $\left(1-a_{p} e^{i \theta}\right)^{-\alpha}$, one sees that for $\alpha$ confined to a compact subset of $\mathbb{C}$,

$$
\frac{1}{\alpha} \int^{\pi}\left|\left(1-a_{p} e^{i \theta}\right)^{-\alpha}\right|^{2} d \theta=1+O\left(\left|a_{p}\right|^{2}\right) .
$$


It follows that $f^{\alpha} \in \mathcal{H}$. In particular, $f^{\gamma N} \in \mathcal{H}$ for all $N=1,2,3, \ldots$, and $\gamma=1,-1, i,-i$. By Corollary 4.3, we have, for each $\varepsilon>0$, and almost all $\chi$,

$$
\left(f_{\chi}(s)\right)^{\gamma}=O\left(\frac{|t|^{\varepsilon}}{\sigma^{\varepsilon}}\right) \quad \text { as } \quad|t| \rightarrow+\infty
$$

uniformly in $\sigma, \sigma>0$, whence the assertion follows.

Corollary 4.10. Fix $\varepsilon>0$. Almost surely in $\chi$,

$$
\log \zeta_{\chi}(\sigma+i t)=o(\log |t|) \quad \text { as } \quad|t| \rightarrow+\infty
$$

holds uniformly in $\sigma, \sigma>\frac{1}{2}+\varepsilon$.

Proof. Apply Theorem 4.9 to the coefficients $a_{n}=n^{-\frac{1}{2}-\delta}$, with $\delta=\frac{1}{2} \varepsilon$.

4.5. Function-theoretic properties of individual functions in $\mathcal{H}$. The purpose of this paragraph is to obtain some basic information about the function theoretic properties of individual functions in $\mathcal{H}$. In particular, we are interested in the structure of their zero sequences. The situation starkly contrasts the "almost sure" behavior that we have concentrated on so far, because here, the functions are only known to be analytic in the half-plane $\Re s>\frac{1}{2}$ (which we denote by $\mathbb{C}_{\frac{1}{2}}$ ), and zeros may actually accumulate at a boundary point such as $s=\frac{1}{2}$.

Let $H_{\infty}^{2}\left(\mathbb{C}_{\frac{1}{2}}\right)$ denote the uniformly local $H^{2}$ space on $\mathbb{C}_{\frac{1}{2}}$ : a function $g$ holomorphic on $\mathbb{C}_{\frac{1}{2}}$ is said to be in it if

$$
\sup _{\theta \in \mathbb{R}} \sup _{\sigma>1 / 2} \int_{\theta}^{\theta+1}|g(\sigma+i t)|^{2} d t<+\infty .
$$

It is a Banach space, and the functions in it are bounded in every half-plane $\Re s>\sigma_{0}$, with $\sigma_{0}>\frac{1}{2}$.

Theorem 4.11. We have the inclusion $\mathcal{H} \subset H_{\infty}^{2}\left(\mathbb{C}_{\frac{1}{2}}\right)$, and the injection mapping is continuous.

Proof. Let $f \in \mathcal{H}$ have the series expansion (2-1). We wish to prove that for all $\sigma>\frac{1}{2}$

$$
\int_{\theta}^{\theta+1}|f(\sigma+i t)|^{2} d t \leq C \sum_{n=1}^{\infty}\left|a_{n}\right|^{2}
$$

holds, where $C$ is an absolute constant. We note that it suffices to obtain (4-8) for finite Dirichlet series $f$, since on compact subsets of $\mathbb{C}_{\frac{1}{2}}$, elements of $\mathcal{H}$ are uniformly approximable by them. Moreover, by making a vertical translation, we see that we can set $\theta=0$, and by the Poisson integral formula, we see that it suffices to consider the limit case $\sigma=\frac{1}{2}$. By duality, we have

$$
\begin{aligned}
\int_{0}^{1}\left|f\left(\frac{1}{2}+i t\right)\right|^{2} d t & =\sup _{g}\left|\int_{0}^{1} \sum_{n} a_{n} n^{-1 / 2} e^{-i t \log n} g(t) d t\right|^{2} \\
& =\sup \left|\sum a_{n} n^{-1 / 2} \widehat{g}(\log n)\right|^{2} \leq \sum^{\infty}\left|a_{n}\right|^{2} \sup \sum^{\infty} n^{-1}|\widehat{g}(\log n)|^{2}
\end{aligned}
$$


where the supremum is taken over all $g \in L^{2}(0,1)$ of norm $\leq 1$, and $\widehat{g}$ is the Fourier transform of $g$,

$$
\widehat{g}(\xi)=\int_{0}^{1} e^{-i t \xi} g(t) d t,
$$

which extends to an entire function of exponential type $\leq 1$. For such functions $\widehat{g}$,

$$
\sum_{n=1}^{\infty} n^{-1}|\widehat{g}(\log n)|^{2} \leq C \int_{-\infty}^{+\infty}|\widehat{g}(\xi)|^{2} d \xi
$$

holds for some absolute constant $C$. This follows from a suitable adaptation of a classical inequality of Plancherel and Pólya [21, pp. 96-98]. Heuristically speaking, the reason is that the left hand side looks like a Riemann sum of part of the integral to the right, and that the functions $\widehat{g}$ are sufficiently smooth. Modulo the Plancherel identity, the assertion now follows from (4-9).

Corollary 4.12. Let $f \in \mathcal{H}, f(s) \not \equiv 0$. If $\left\{s_{k}\right\}_{k}, s_{k}=\sigma_{k}+i t_{k}$, denotes the sequence of zeros of $f$ in $\mathbb{C}_{\frac{1}{2}}$, then

$$
\sup _{\theta \in \mathbb{R}} \sum_{k: s_{k} \in Q_{\theta}}\left(\sigma_{k}-\frac{1}{2}\right)<+\infty
$$

where $Q_{\theta}$ is the half-strip $\Re s>0, \theta<\Im s<\theta+1$.

Proof. By Theorem 4.11, $f(s) / s$ is contained in $H^{2}\left(\mathbb{C}_{\frac{1}{2}}\right)$, and so $\left\{s_{k}\right\}_{k}$ satisfies the Blaschke condition

$$
\sum_{k} \frac{\sigma_{k}-\frac{1}{2}}{1+\left|s_{k}\right|^{2}}<+\infty .
$$

All convergent nontrivial Dirichlet series have a zero-free half-plane, so that

$$
\sup _{k} \sigma_{k}<+\infty
$$

Hence

$$
A(\theta)=\sum_{k: s_{k} \in Q_{\theta}}\left(\sigma_{k}-\frac{1}{2}\right)<+\infty,
$$

for each real $\theta$. If the function $A(\theta)$ were unbounded, we could find a sequence $\theta_{k}$ of reals such that $A\left(\theta_{k}\right) \rightarrow+\infty$. But then the functions $f_{k}(s)=f\left(s+i \theta_{k}\right)$ would tend to 0 uniformly on compact subsets of $\mathbb{C}_{\frac{1}{2}}$, because they would have an ever increasing mass of zeros, and their norms in $H_{\infty}^{2}\left(\mathbb{C}_{\frac{1}{2}}\right)$ are uniformly bounded. By Lemma 2.4, any vertical limit function has the form

$$
\sum_{n=1}^{\infty} a_{n} \chi(n) n^{-s}
$$

where $\chi$ is a character. Such a function cannot be identically zero. This is a contradiction, and so $A(\theta)$ is bounded. 
Proposition 4.13. There exists a function $f \in \mathcal{H}$, other than the 0 function, whose zero set contains a subsequence tending to $s=\frac{1}{2}$ along the real line.

Proof. Let $\left\{b_{n}\right\}_{n}$ be the sequence $b_{1}=0, b_{n}=n^{-1 / 2}(\log n)^{-1}$ for $n>1$, and notice that it has $\sum_{n} b_{n}^{2}<+\infty$ and $\sum_{n} n^{-1 / 2} b_{n}=+\infty$. We now select integers $n_{k}$ and points $\sigma_{k}$ according to the following fashion. Let $n_{0}=1$. Given $n_{k}$, we pick $\sigma_{k}>\frac{1}{2}$ so close to $\frac{1}{2}$ and $n_{k+1}$ so much larger than $n_{k}$ that

$$
\sum_{n=n_{k}}^{n_{k+1}-1} n^{-\sigma_{k}} b_{n}-\sum_{n=1}^{n_{k}-1} n^{-\sigma_{k}} b_{n}-\sum_{n=n_{k+1}}^{\infty} n^{-\sigma_{k}} b_{n}>0
$$

The sequence $\left\{\sigma_{k}\right\}_{k}$ is strictly decreasing, with limit $\frac{1}{2}$. If we put $a_{n}=(-1)^{k} b_{n}$ for $n_{k} \leq n<n_{k+1}$, and let $f$ be as in (2-1), then $f$ is real-valued on the real half-axis $] \frac{1}{2},+\infty\left[\right.$, and the sign of $f\left(\sigma_{k}\right)$ alternates as $(-1)^{k}$. By the intermediate value theorem of calculus, $f$ has infinitely many zeros along the real line, which accumulate at $s=\frac{1}{2}$.

\section{Systems of Dilated FunCtions in $L^{2}(0,1)$}

In this section, we return to the basis and completeness problems described in the introduction.

Let $\varphi \in L^{2}(0,1)$ be given and again consider it as an odd periodic function of period 2. The basic questions are: For which $\varphi$ is the system

$$
\varphi(x), \varphi(2 x), \varphi(3 x), \ldots
$$

a Riesz basis in $L^{2}(0,1)$, and for which $\varphi$ is the same system complete in $L^{2}(0,1)$ ? We recall that completeness means that the system spans a dense linear subspace. The observation that the only orthogonal bases of this kind are generated by the functions $\varphi(x)=C \sin (\pi x)$ motivates introducing the representation

$$
\varphi(x)=\sum_{n=1}^{\infty} a_{n} \sqrt{2} \sin (n \pi x)
$$

for an arbitrary $\varphi \in L^{2}(0,1)$, and seeking solutions in terms of the coefficients $a_{n}$. For both problems, a necessary condition is $a_{1} \neq 0$, because otherwise the function $\sin (\pi x)$ cannot be approximated. It is no restriction to normalize: $a_{1}=1$ from now on.

Let $\left\{e_{n}\right\}_{n}$ be the standard orthonormal basis $e_{n}(x)=\sqrt{2} \sin (n \pi x)$. Following Beurling [1], we associate to each $f \in L^{2}(0,1)$ with sine series expansion $f(x)=$ $\sum_{n} c_{n} e_{n}(x)$ the auxiliary Dirichlet series

$$
S f(s)=\sum_{n=1}^{\infty} c_{n} n^{-s}
$$

Let $\|\cdot\|_{L^{2}}$ and $\langle\cdot, \cdot\rangle_{L^{2}}$ be the norm and inner product in $L^{2}(0,1)$, respectively. The operator $S$ is an isometric isomorphism between $L^{2}(0,1)$ and $\mathcal{H}:\|S f\|_{\mathcal{H}}=\|f\|_{L^{2}}$. 
$L^{2}(0,1)$ of the form $f(x)=\sum_{n} c_{n} e_{n}(x)$, where only finitely many $c_{n}$ are nonzero, we associate to it the function $T_{\varphi} f(x)=\sum_{n} c_{n} \varphi_{n}(x)$, where $\varphi_{n}(x)=\varphi(n x)$. It is clear that $T_{\varphi} f \in L^{2}(0,1)$, given the restriction on $f$. When the operator $S$ is applied to $T_{\varphi} f$, we arrive at the identity

$$
S\left(T_{\varphi} f\right)(s)=S \varphi(s) S f(s), \quad \Re s>\frac{1}{2} .
$$

This has the interpretation that replacing the basis sequence $\left\{e_{n}\right\}_{n}$ with $\left\{\varphi_{n}\right\}_{n}$ corresponds to multiplication by $S \varphi$ on the $S$-transformed side.

5.1 The function $1 / S \varphi$. The biorthogonal system. Let $S \varphi(s)=\sum_{n} a_{n} n^{-s}$, as before. We formally write $1 / S \varphi(s)=\sum_{n} b_{n} n^{-s}$. The coefficients $b_{n}$ are given by $b_{1}=1$,

$$
b_{n}=\sum_{d_{1} d_{2} \cdots d_{k}=n}(-1)^{k} a_{d_{1}} a_{d_{2}} \cdots a_{d_{k}}, \quad n>1
$$

where the sum runs over all the finitely many possible product decompositions of $n$, where the various factors $d_{j}$ are integers $>1$. The series $\sum_{n} b_{n} n^{-s}$ converges absolutely in some half-plane $\Re s>\sigma_{0}$, as is readily seen by considering the extremal situation where $a_{n} \leq 0$ for all $n>1$. In fact, any $\sigma_{0}$ with $\sum_{n>1}\left|a_{n}\right| n^{-\sigma_{0}} \leq a_{1}=1$ will do. Multiplying $S \varphi$ with $1 / S \varphi$, we read off from the coefficients that $b_{1} a_{1}=1$ and

$$
\sum_{d: d \mid n} b_{d} a_{n / d}=0, \quad n>1 .
$$

Formally, we have $1 / S \varphi=S \psi$, where $\psi(x)=\sum_{n=1}^{\infty} b_{n} e_{n}(x)$. It follows from (5-2) that the functions $\psi_{n}(x)=\sum_{d: d \mid n} \bar{b}_{n / d} e_{d}(x)$ (they are not the dilates of $\psi$ ) form a system which is biorthogonal to the original system $\left\{\varphi_{n}\right\}_{n}$, with $\varphi_{n}(x)=\varphi(n x)$, that is, $\left\langle\varphi_{j}, \psi_{k}\right\rangle=\delta_{j, k}$, where $\delta_{j, k}$ is the Kronecker delta symbol, which is 1 when $j=k$, and 0 otherwise. It follows that the system $\left\{\varphi_{n}\right\}_{n}$ is minimal, that is, each $\varphi_{n}$ lies outside the closure of the linear span of the other vectors. Moreover, $e_{n}(x)=\sum_{d: d \mid n} a_{n / d} \psi_{d}(x)$, and since these sums contain only a finite number of terms, it is clear that the biorthogonal system $\left\{\psi_{n}\right\}_{n}$ is complete in $L^{2}(0,1)$. The biorthogonal system consists of dilations of a single function only in the trivial case $\varphi(x)=e_{1}(x)=\sqrt{2} \sin (\pi x)$.

5.2. Riesz bases. Recall that a basis $\left\{f_{n}\right\}_{n}$ in a separable Hilbert space $H$ is a Riesz basis if for some bounded invertible operator $L$ on $H$, the sequence $\left\{L f_{n}\right\}_{n}$ is an orthonormal basis. An equivalent characterization is the following (see [21, pp. 30-37]).

Lemma 5.1. Let $H$ be a separable Hilbert space. A system $\left\{f_{n}\right\}_{n}$ of vectors from $H$ is a Riesz basis in $H$ if and only if

(a) every $f \in H$ can be expanded as $f=\sum_{n} c_{n} f_{n}$.

(b) there are constants $A$ and $B, 0<A \leq B<+\infty$, such that

$$
A\left(\sum_{n}\left|c_{n}\right|^{2}\right)^{1 / 2} \leq\left\|\sum_{n} c_{n} f_{n}\right\|_{L^{2}} \leq B\left(\sum_{n}\left|c_{n}\right|^{2}\right)^{1 / 2}
$$

for every finite sequence of scalars $c_{n}$. 
Theorem 5.2. The system $\varphi(x), \varphi(2 x), \varphi(3 x), \ldots$ is a Riesz basis in $L^{2}(0,1)$ if and only if both $S \varphi$ and $1 / S \varphi$ belong to $\mathcal{M}$.

Proof. As before, we write $e_{n}(x)=\sqrt{2} \sin (n \pi x)$, and let $T_{\varphi}$ be the linear mapping which sends $e_{n}$ to $\varphi_{n}$, for $n=1,2,3, \ldots$ Let $\mathfrak{F}$ denote the dense subspace of $L^{2}(0,1)$ consisting of functions $f=\sum_{n} c_{n} e_{n}$, where all but finitely many of the coefficients $c_{n}$ equal 0. By Lemma 5.1, we have a Riesz basis if and only if the image of $\mathfrak{F}$ under $T_{\varphi}$ is dense in $L^{2}(0,1)$, and

$$
A\|f\|_{L^{2}} \leq\left\|T_{\varphi} f\right\|_{L^{2}} \leq B\|f\|_{L^{2}}, \quad f \in \mathfrak{F},
$$

holds for some constants $A$ and $B, 0<A \leq B<+\infty$. After an application of the transformation $S$, the Riesz basis condition becomes, if we use (5-1), that the image of $S \mathfrak{F}$ under multiplication by $S \varphi$ should be dense in $\mathcal{H}$, and that

$$
A\|S f\|_{\mathcal{H}} \leq\|S \varphi S f\|_{\mathcal{H}} \leq B\|S f\|_{\mathcal{H}}, \quad f \in \mathfrak{F} .
$$

We first do the sufficiency part. If $S \varphi$ and $1 / S \varphi$ are both in $\mathcal{M}$, then clearly, $S \varphi S \mathfrak{F}$ is dense, and (5-5) holds with $A=\|1 / S \varphi\|_{\mathcal{M}}^{-1}$ and $B=\|S \varphi\|_{\mathcal{M}}$.

We turn to the necessity part. By an argument involving Cauchy sequences, (5-4) extends to all $f \in L^{2}(0,1)$, so that $S \varphi$ is a multiplier on $\mathcal{H}$. By $(5-5)$ and the fact that $S \varphi S \mathfrak{F}$ is dense in $\mathcal{H}, 1 / S \varphi$ is a multiplier on $\mathcal{H}$ as well. The proof is complete.

Example. In conjunction with Theorem 3.1, the theorem above provides a precise statement about the subtle dependence on the "closeness" of $\varphi$ to $e_{1}(x)=$ $\sqrt{2} \sin (\pi x)$. To illuminate the condition, consider

$$
\varphi(x)=\sum_{n=1}^{\infty} \frac{e_{n}(x)}{n^{\tau}}=\sqrt{2} \sum_{n=1}^{\infty} \frac{\sin (n \pi x)}{n^{\tau}},
$$

for $\tau>\frac{1}{2}$. Then

$$
S \varphi(s)=\sum_{n=1}^{\infty} n^{-\tau} n^{-s}=\zeta(\tau+s),
$$

and

$$
1 / S \varphi(s)=\sum_{n=1}^{\infty} \mu(n) n^{-\tau} n^{-s},
$$

where $\mu(n)$ is the Möbius function, which has $\mu(1)=1, \mu(n)=(-1)^{k}$ when $n$ is the product of $k$ different primes, and $\mu(n)=0$ if $n$ is divisible by a square (other than 1). The function $S \varphi(s)$ is bounded in the half-plane $\Re s>0$ if and only if $\tau>1$. Using Theorem 5.2 and the fact that multipliers are bounded analytic functions, we conclude that the system $\varphi(x), \varphi(2 x), \ldots$ is a Riesz basis in $L^{2}(0,1)$ if and only if $\tau>1$. 
Corollary 5.3. If the coefficients $a_{n}$ of $S \varphi \in \mathcal{H}$ are totally multiplicative, the functions $\varphi(x), \varphi(2 x), \varphi(3 x), \ldots$ form a Riesz basis in $L^{2}(0,1)$ if and only if $\sum_{p}\left|a_{p}\right|<$ $+\infty$, where the sum runs over the primes.

Proof. Since the totally multiplicative coefficients come from a function in $\mathcal{H}$, they must satisfy $\sup _{n}\left|a_{n}\right|<1$, and by the Euler product formula,

$$
S \varphi(s)=\sum_{n=1}^{\infty} a_{n} n^{-s}=\prod_{p}\left(1-a_{p} p^{-s}\right)^{-1} .
$$

By the remark following the proof of the multiplier theorem, $\mathcal{M}$ is isometrically isomorphic to $H^{\infty}\left(\mathbb{D}^{\infty}\right)$. As we calculate the norm of $\mathfrak{Q} S \varphi$ there, using the analog of (5-6), we obtain

$$
\|S \varphi\|_{\mathcal{M}}=\prod_{p}\left(1-\left|a_{p}\right|\right)^{-1}
$$

so that in view of Theorem $5.2, \sum_{p}\left|a_{p}\right|<+\infty$ is certainly a necessary condition to have a Riesz basis. Incidentally, this is also sufficient, since

$$
1 / S \varphi(s)=\sum_{n=1}^{\infty} \mu(n) a_{n} n^{-s}=\prod_{p}\left(1-a_{p} p^{-s}\right)
$$

Note next the following consequence of Theorems 3.1 and 5.2; for the second statement, one should keep in mind the identification of $\mathcal{M}$ with $H^{\infty}\left(\mathbb{D}^{\infty}\right)$ supplied by Section 3.

Corollary 5.4. If $\varphi(x)=\sum_{n} a_{n} e_{n}(x)$, and $\sum_{n>1}\left|a_{n}\right|<a_{1}=1$, then the collection $\{\varphi(n x)\}_{n}(n=1,2,3, \ldots)$ forms a Riesz basis in $L^{2}(0,1)$. On the other hand, if $a_{n}=0$ unless $n$ is a prime or 1 , the condition $\sum_{n=2}^{\infty}\left|a_{n}\right|<1$ is necessary to have a Riesz basis.

5.3. The completeness problem. The completeness problem is considerably more delicate than the Riesz basis problem. It does not seem likely that it has a solution as simple as the one given by Theorem 5.2. In fact, as we shall see, it is equivalent to the problem of describing the cyclic vectors in the space $H^{2}\left(\mathbb{D}^{\infty}\right)$, which is definitely quite hard. The relationship to the invariant subspaces in an infinite-dimensional setting was pointed out by the editors of Beurling's collected works [1]. The level of difficulty of Beurling's completeness problem is emphasized by the fact that a complete characterization of the cyclic vectors in $H^{2}\left(\mathbb{D}^{N}\right)$ is known only when the (complex) dimension of the polydisk is $N=1$.

A subspace $I$ of $\mathcal{H}$ is said to be invariant if it is closed, and $f g \in I$ whenever $f \in I$ and $g \in \mathfrak{G}$. One shows, by suitably approximating functions in $\mathcal{M}$ with elements of $\mathfrak{G}$, that invariant subspaces are actually invariant under multiplication by elements of $\mathcal{M}$. The invariant subspace generated by a function $f \in \mathcal{H}$ is denoted by $[f]_{\mathcal{H}}$, and we say that $f$ is cyclic if $[f]_{\mathcal{H}}=\mathcal{H}$. Similarly, a subspace $J$ of $H^{2}\left(\mathbb{D}^{\infty}\right)$ is called invariant if it is closed, and invariant under multiplication by polynomials in the coordinate functions $z_{1}, z_{2}, \ldots$ on $\mathbb{D}^{\infty}$. The invariant subspaces are actually invariant under multiplication by $H^{\infty}\left(\mathbb{D}^{\infty}\right)$. We write $[f]_{H^{2}(\mathbb{D} \infty)}$ for the invariant 
A moment's reflection on the definition of $T_{\varphi}$ reveals that $\varphi_{1}, \varphi_{2}, \varphi_{3}, \ldots$ form a complete system in $L^{2}(0,1)$ if and only if the image of $\mathfrak{F}$ under $T_{\varphi}$ is dense in $L^{2}(0,1)$. After an application of the transformation $S$, using (5-1), the latter condition becomes the requirement that $S \varphi$ times the set $\mathfrak{G}=S \mathfrak{F}$ of finite Dirichlet series be dense in $\mathcal{H}$, id est, that $S \varphi$ be cyclic in $\mathcal{H}$. By the isometry between $\mathcal{H}$ and $H^{2}\left(\mathbb{D}^{\infty}\right)$ supplied by the operator $\mathfrak{Q}$ back in Section 2, the following can be said.

Theorem 5.5. Let $\varphi$ be as above. Then the following are equivalent:

(a) the system $\varphi_{1}, \varphi_{2}, \varphi_{3}, \ldots$ is complete in $L^{2}(0,1)$.

(b) the function $S \varphi$ is cyclic in $\mathcal{H}$, that is, the subspace $S \varphi \mathcal{M}$ is dense in $\mathcal{H}$.

(c) the function $\mathfrak{Q} S \varphi$ is cyclic in $H^{2}\left(\mathbb{D}^{\infty}\right)$.

A necessary condition for the completeness of the system $\left\{\varphi_{n}\right\}_{n}$ mentioned by Beurling in his 1945 seminar (confer [1, p. 378]), is that the function $S \varphi(s)$ be zerofree in the half-plane $\Re s>\frac{1}{2}$. We shall prove something slightly stronger. For a sequence $z=\left(z_{j}\right)_{j}$ in $\mathbb{D}^{\infty}$, let $\|z\|_{l^{\infty}}=\sup \left\{\left|z_{j}\right|: j=1,2, \ldots\right\}$ and $\|z\|_{l^{2}}^{2}=\sum_{j}\left|z_{j}\right|^{2}$. We extend this notation to sets $\Omega \in \mathbb{D}^{\infty}$ by taking suprema over all the points in $\Omega$.

Lemma 5.6. If the system $\varphi_{1}, \varphi_{2}, \ldots$ is complete in $L^{2}(0,1)$, and $\Omega \subset \mathbb{D}^{\infty}$ has $\|\Omega\|_{l^{\infty}}<1$ and $\|\Omega\|_{l^{2}}<+\infty$, then

$$
\inf _{z \in \Omega}|\mathfrak{Q} S \varphi(z)|>0
$$

Proof. In view of Theorem 5.5, we need to show that if $g \in H^{2}\left(\mathbb{D}^{\infty}\right)$ is cyclic, then $|g|$ is bounded away from 0 on $\Omega$. We argue by contradiction. So, suppose $|g|$ is not bounded away from 0 on $\Omega$; then there is a sequence of points $z(k)$ in $\Omega$ such that $g(z(k)) \rightarrow 0$ as $k \rightarrow+\infty$. When $l^{2}(\mathbb{N})$ is given the weak topology, its closed unit ball becomes a compact metric space (the Banach-Alaoglu theorem), so that by the Bolzano-Weierstraß theorem, the sequence $\{z(k)\}_{k}$ possesses a cluster point $z(\infty)$, with $\|z(\infty)\|_{l^{\infty}}<1$ and $\|z(\infty)\|_{l^{2}}<+\infty$. By the conditions on $\Omega, \sup _{z \in \Omega} \prod_{j}\left(1-\left|z_{j}\right|^{2}\right)^{-1}<+\infty$, so that point evaluations in $\Omega$ are uniformly bounded. It follows that as the subsequence of points $z\left(k_{l}\right)$ converges to $z(\infty)$, the function values $g\left(z\left(k_{l}\right)\right)$ converge to $g(z(\infty))$, so that $g(z(\infty))=0$. We conclude that $g$ cannot be cyclic, as it is annihilated by a bounded point evaluation.

Remark. In particular, it follows that for the system $\varphi_{1}, \varphi_{2}, \ldots$ to be complete, a necessary condition is that

$$
\sup _{\Re s>\sigma_{0}}|S \varphi(s)|>0
$$

for any $\sigma_{0}>\frac{1}{2}$. This result is sharp, in the sense that for complete systems, $\inf _{\Re s>\frac{1}{2}}|S \varphi(s)|^{2}=0$ can occur. An example is given after Corollary 5.8. A slightly more refined necessary condition for completeness can be obtained from Theorem 4.10: for all characters $\chi$, the function $(S \varphi)_{\chi}(s)=\sum_{n} a_{n} \chi(n) n^{-s}$ must be outer in $H_{\infty}^{2}\left(\mathbb{C}_{\frac{1}{2}}\right)$. Also, by a remark Helson makes in $[\mathbf{1 6}]$, it is necessary that $(S \varphi)_{\chi}$ almost surely be outer in $H_{\mathrm{i}}^{2}\left(\mathbb{C}_{+}\right)$. 
5.4. The Dirichlet-type space $\mathcal{H}_{d}$. We introduce next a subspace of $\mathcal{H}$ which seems to play a natural role in the study of the completeness problem. To this end, let

$$
d(n)=\sum_{d: d \mid n} 1
$$

denote the number of divisors of a natural number $n$. As a function of $n$, the number of divisors is quite irregular, but it is well-known that $d(n)=O\left(n^{\delta}\right)$ holds for every $\delta>0$; however, the average order of $d(n)$ is $\log n$, confer [13, pp. 260-266]. We denote by $\mathcal{H}_{d}$ the collection of Dirichlet series $f(s)=\sum_{n=1}^{\infty} a_{n} n^{-s}$ for which

$$
\sum_{n=1}^{\infty}\left|a_{n}\right|^{2} d(n)<\infty .
$$

The set of those $f$ in $\mathcal{H}_{d}$ for which $a_{n}=0$ unless $n$ is a power of a given prime $p$, coincides with the usual Dirichlet space on the unit disk, if we set $z=p^{-s}$.

Clearly, $\mathcal{H}_{d} \subset \mathcal{H}$. For the interesting class of series with totally multiplicative coefficients $\left(a_{m n}=a_{m} a_{n}\right)$, we have the opposite inclusion. Indeed,

$$
\sum_{n}\left|a_{n}\right|^{2} d(n)=\left(\sum_{n}\left|a_{n}\right|^{2}\right)^{2}
$$

in this favorable case. The identity can be verified by expanding both sums as Euler products. If we write $n=\prod_{p} p^{\nu_{p}}$, where the product runs over the primes, and all but finitely many of the nonnegative integers $\nu_{p}$ are 0 , we have

$$
\begin{gathered}
\sum_{n}\left|a_{n}\right|^{2} d(n)=\sum_{n}\left|a_{2}^{\nu_{2}} a_{3}^{\nu_{3}} \cdots a_{p}^{\nu_{p}} \cdots\right|^{2}\left(1+\nu_{2}\right)\left(1+\nu_{3}\right) \cdots\left(1+\nu_{p}\right) \cdots \\
=\sum_{\nu_{2}=0}^{\infty}\left(1+\nu_{2}\right)\left|a_{2}\right|^{2 \nu_{2}} \cdot \sum_{\nu_{3}=0}^{\infty}\left(1+\nu_{3}\right)\left|a_{3}\right|^{2 \nu_{3}} \cdots \sum_{\nu_{p}=0}^{\infty}\left(1+\nu_{p}\right)\left|a_{p}\right|^{2 \nu_{p}} \cdots \\
=\left(1-\left|a_{2}\right|^{2}\right)^{-2} \cdot\left(1-\left|a_{3}\right|^{2}\right)^{-2} \cdots\left(1-\left|a_{p}\right|^{2}\right)^{-2} \cdots=\prod_{p}\left(1-\left|a_{p}\right|^{2}\right)^{-2}
\end{gathered}
$$

provided that each $\left|a_{p}\right|<1$, and $a_{1}=1$. Note that if some $\left|a_{p}\right| \geq 1$, the sequence $\left\{a_{n}\right\}_{n}$ is not even in $l^{2}(\mathbb{N})$. We have, by using a similar argument,

$$
\sum_{n=1}^{\infty}\left|a_{n}\right|^{2}=\prod_{p}\left(1-\left|a_{p}\right|^{2}\right)^{-1}
$$

where $a_{1}=1$ and $\left|a_{p}\right|<1$. The product converges if and only if

$$
\sum_{p}\left|a_{p}\right|^{2}<+\infty, \quad\left|a_{p}\right|<1
$$

This is a necessary and sufficient criterion for a Dirichlet series with totally multi- 
The reciprocal series of a Dirichlet series $f(s)=\sum_{n} a_{n} n^{-s}$ with totally multiplicative coefficients and $a_{1}=1$ is

$$
\frac{1}{f(s)}=\sum_{n=1}^{\infty} \mu(n) a_{n} n^{-s}
$$

where $\mu(n)$ is the Möbius function. A direct calculation yields

$$
\sum_{n=1}^{\infty}\left|\mu(n) a_{n}\right|^{2}=\prod_{p}\left(1+\left|a_{p}\right|^{2}\right), \quad \sum_{n}\left|\mu(n) a_{n}\right|^{2} d(n)=\prod_{p}\left(1+2\left|a_{p}\right|^{2}\right)
$$

for the expressions defining the norms in $\mathcal{H}$ and in $\mathcal{H}_{d}$. These norms are finite if and only if $\sum_{p}\left|a_{p}\right|^{2}<+\infty$. This criterion for $1 / f(s)$ differs from the one for $f(s)$ in one respect: the condition $\left|a_{p}\right|<1$ is skipped.

5.5 Completeness, continued. We now use the space $\mathcal{H}_{d}$ to formulate a fairly general sufficient condition for completeness.

Theorem 5.7. The system $\left\{\varphi_{n}\right\}_{n}$ is complete in $L^{2}(0,1)$ if one of the following conditions is fulfilled:

(a) $S \varphi$ and $1 / S \varphi$ are both in $\mathcal{H}_{d}$.

(b) $S \varphi \in \mathcal{M}$ and $1 / S \varphi \in \mathcal{H}$.

(c) $S \varphi \in \mathcal{H}$ and $1 / S \varphi \in \mathcal{M}$.

Proof. We first look at parts (a) and (b). As before, we write $1 / S \varphi(s)=\sum_{n} b_{n} n^{-s}$. Put $R_{N}(s)=\sum_{n=1}^{N} b_{n} n^{-s}$. Suppose we can show that $\left\|S \varphi R_{N}\right\|_{\mathcal{H}}$ has a bound which is independent of $N$. Then $S \varphi R_{N}$ tends to the constant function 1 weakly, as $N \rightarrow+\infty$. By standard functional analysis, 1 is in the norm closure of $S \varphi \mathcal{M}$, so that $S \varphi$ is cyclic in $\mathcal{H}$. Under condition (b), it is clear that this boundedness condition is fulfilled, by the estimate $\left\|R_{N}\right\|_{\mathcal{H}} \leq\|1 / S \varphi\|_{\mathcal{H}}$. Under condition (a), we apply the Cauchy-Schwarz inequality,

$$
\begin{aligned}
\left\|S \varphi R_{N}\right\|_{\mathcal{H}}^{2}=\sum_{k} \mid & \left.\sum_{\substack{m, n: \\
m n=k, m \leq N}} a_{n} b_{m}\right|^{2} \leq \sum_{k} d(k) \sum_{m, n: m n=k}\left|a_{n}\right|^{2}\left|b_{m}\right|^{2} \\
& \leq\left(\sum_{n}\left|a_{n}\right|^{2} d(n)\right)\left(\sum_{m}\left|b_{m}\right|^{2} d(m)\right)=\|S \varphi\|_{\mathcal{H}}^{2}\|1 / S \varphi\|_{\mathcal{H}}^{2},
\end{aligned}
$$

where we used that $d(m n) \leq d(m) d(n)$. Part (a) follows. Under condition (c), we just multiply $S \varphi$ with the function $1 / S \varphi$ in $\mathcal{M}$, to get that $1 \in[S \varphi]_{\mathcal{H}}$, so that $S \varphi$ is cyclic. The proof is complete.

Corollary 5.8. If the coefficients of $S \varphi \in \mathcal{H}$ are totally multiplicative, the system $\left\{\varphi_{n}\right\}_{n}$ is complete in $L^{2}(0,1)$.

Proof. According to the previous subsection, we have that both $S \varphi$ and $1 / S \varphi$ are in $\mathcal{H}_{d}$, because the coefficients are totally multiplicative. The assertion now follows from part (a) of Theorem 5.7.

Remark. It follows that in the context of the example considered after Theorem 5.2 , we have completeness if and only if $\tau>\frac{1}{2}$. In a way, this reproves a theorem 
Example. We use Theorem 5.7 to construct a complete system $\varphi_{1}, \varphi_{2}, \ldots$ with

$$
\inf _{\Re s>\frac{1}{2}}|S \varphi(s)|=0,
$$

as promised in the remark following Lemma 5.6. Let $b_{1}=1, b_{2}=0$, and put $b_{p}=$ $p^{-1 / 2}(\log \log p)^{2 / 3}$ for prime indices $p>2$, and extend the sequence multiplicatively, so that $b_{m n}=b_{m} b_{n}$. By Chebyshev's theorem [19, Vol. 1, p. 25], the series

$$
\sum_{p}\left|b_{p}\right|^{2}=\sum_{p: p>2} \frac{1}{p(\log \log p)^{4 / 3}}
$$

(summation over the primes) converges if and only if

$$
\sum_{n=3}^{\infty} \frac{1}{n(\log \log n)^{4 / 3} \log n}
$$

converges. Since the last series converges by the integral test, we conclude from the results of the previous subsection that the functions $\Phi$ and $1 / \Phi$ are in $\mathcal{H}_{d}$, where $\Phi(s)=\sum_{n} b_{n} n^{-s}$. Let $\varphi$ be given by $S \varphi=1 / \Phi$. The completeness now follows from Theorem 5.7. Again by Chebyshev's theorem,

$$
\Phi(\sigma) \geq \sum_{p: p>2} \frac{1}{p^{1 / 2+\sigma}(\log \log p)^{2 / 3}} \geq A \sum_{n=3}^{\infty} \frac{1}{n^{1 / 2+\sigma}(\log \log n)^{2 / 3} \log n},
$$

for some positive absolute constant $A$, whence $\Phi(\sigma) \rightarrow+\infty$ as $\sigma \rightarrow \frac{1}{2}$, since the right hand side series diverges for $\sigma=\frac{1}{2}$. It follows that $S \varphi(\sigma) \rightarrow 0$ as $\sigma \rightarrow \frac{1}{2}$.

Theorem 5.9. Let $S \varphi(s)=1+\sum_{p} a_{p} p^{-s}$, summing only over the primes. Then the system $\left\{\varphi_{n}\right\}_{n}$ is complete in $L^{2}(0,1)$ if and only if $\sum_{p}\left|a_{p}\right| \leq 1$.

Proof. To see the necessity, observe that if $\sum_{p}\left|a_{p}\right|>1$, there exist a positive integer $N$ and and a point $z=\left(z_{1}, z_{2}, \ldots\right) \in \mathbb{D}^{\infty}$ with $z_{j}=0$ for $j>N$, such that $\left(p_{j}\right.$ is the $j$-th prime)

$$
\mathfrak{Q} S \varphi(z)=1+\sum_{j=1}^{\infty} a_{p_{j}} z_{j}=0 .
$$

By Lemma 5.6, we cannot have completeness.

To get the sufficiency, we introduce the auxiliary function $g=1-S \varphi$, and note that $\|g\|_{\mathcal{M}} \leq 1$. The function $1-g^{n}=(1-g)\left(1+g+\ldots+g^{n-1}\right)$ tends to 1 in the norm of $\mathcal{H}$ as $n \rightarrow+\infty$, and for each $n$, it is in $S \varphi \mathcal{M}$. It follows that $S \varphi$ is cyclic.

\section{Possible directions of further investigation}

According to a theorem of Helson (Theorem 4.4), we have that for $f \in \mathcal{H}$, with series expansion (2-1), the Dirichlet series

$$
f_{\chi}(s)=\sum^{\infty} a_{n} \chi(n) n^{-s}
$$


converges in the half-plane $\Re s>0$, for almost all characters $\chi$. In terms of the coefficients, this amounts to having, almost surely in $\chi$,

$$
\sum_{n=1}^{N} a_{n} \chi(n)=O\left(N^{\varepsilon}\right) \quad \text { as } \quad N \rightarrow+\infty
$$

for each fixed $\varepsilon>0$. In view of Theorem 4.5 and Lennart Carleson's theorem on the almost everywhere convergence of Fourier series with $l^{2}$ coefficients [6], which cover the probabilistic and deterministic extremes, the following conjecture seems reasonable: for almost all $\chi$,

$$
\sum_{n=1}^{N} a_{n} \chi(n)=O(1) \quad \text { as } \quad N \rightarrow+\infty .
$$

The conjecture appears to be related to the question of how many of the stochastic variables $\chi(n)$ on a given interval $N \leq n<N+k$ are mutually independent. The latter translates into the following basic number-theoretic problem: What is the multiplicatively rational dimension of the interval $N \leq n<N+k$ ? Here, the multiplicatively rational dimension of a set $E$ of positive integers is the dimension of the $\mathbb{Q}$-linear span of $\log E$ over the field $\mathbb{Q}$. For instance, the multiplicatively rational dimension of the interval $1 \leq n \leq k$ equals the number of primes $\leq k$.

By Corollary 4.7, almost every $\zeta_{\chi}(s)$ converges to a zero-free analytic function in the half-plane $\Re s>\frac{1}{2}$. It will now be indicated how this result may be used to obtain information about particular characters. Given positive reals $M$ and $\varepsilon$, let $\Omega(\varepsilon, M)$ be the set of all $\chi$ for which

$$
\left|\sum_{n=1}^{N} \chi(n)\right| \leq M \cdot N^{1 / 2+\varepsilon}
$$

for all $N=1,2,3, \ldots$ By Corollary 4.8, the $\rho$-mass of $\Omega(\varepsilon, M)$ tends to 1 as $M \rightarrow+\infty$, for fixed $\varepsilon$. A point $\chi_{0}$ in $\Xi$ is a mass point for $\Omega(\varepsilon, M)$ provided that each open neighborhood of $\chi_{0}$, intersected with $\Omega(\varepsilon, M)$, has positive $\rho$-mass. If $\chi_{0}$ is a mass point for some $\Omega(\varepsilon, M)$, then $\zeta_{\chi_{0}}$ extends analytically to a zero-free function on $\Re s>\frac{1}{2}+\varepsilon$. This is so because this function may be approximated by functions $\zeta_{\chi}(s)$ which lack zeros on $\Re s>\frac{1}{2}$, in the topology of uniform convergence on compact subsets of $\Re s>\frac{1}{2}+\varepsilon$. This may be a way to handle non-principal Dirichlet characters, a topic to be discussed below.

As a matter of definition, a character is a multiplicative mapping $\chi: \mathbb{Q}_{+} \rightarrow \mathbb{T}$, and if we like, we may extend it to all of $\mathbb{Q} \backslash\{0\}$ by setting $\chi(-1)$ equal to 1 or -1 . The characters that so extend continuously to the archimedian completion $\mathbb{R} \backslash\{0\}$ are of the type $\chi(r)=|r|^{-i t}$ for some real number $t$ (if we fix $\chi(-1)=1$ ). There are also the non-archimedian completions $\mathbb{Q}_{p}$, the $p$-adic number field, for a given fixed prime $p$. The Dirichlet characters associated with $\mathbb{Q}_{p}$ are the ones that extend continuously to $\mathbb{Q}_{p} \backslash\{0\}$, and one checks that for some $k \in \mathbb{N}$, when restricted to the integers, they are periodic with period $p^{k}$, except on numbers divisible by $p$. Traditionally, one sets the value of the Dirichlet character $\chi$ equal to 0 on the integers divisible by $p$, but this is not our choice here. The principal 
because we do not prescribe the value at $p$ ). The nonprincipal Dirichlet characters all have partial sums $\sum_{n: n \leq N} \chi(n)$ that grow like $O(\log N)$ as $N$ tends to infinity (with the traditional definition, these sums are actually bounded), so at first glance they seem like ideal candidates for mass points. In the final analysis, this may prove to be naïve.

Acknowledgement. The first author wishes to thank Paul Cohen (Stanford) for helpful conversations at an early stage of this work.

\section{REFERENCES}

1. A. Beurling, The Collected Works of Arne Beurling, Volume 2: Harmonic Analysis, Birkhäuser, Boston, 1989, pp. 378-380.

2. H. F. Bohnenblust and E. Hille, On the absolute convergence of Dirichlet series, Ann. Math. 32 (1931), 600-622.

3. H. Bohr, Über die gleichmässige Konvergenz Dirichletscher Reihen, J. Reine Angew. Math. 143 (1913), 203-211.

4. H. Bohr, Über die Bedeutung der Potenzreihen unendlich vieler Variabeln in der Theorie der Dirichletschen reihen $\sum a_{n} / n^{s}$, Nachr. Ges. Wiss. Göttingen Math. Phys. Kl. (1913), 441-488.

5. H. Bohr, Harald Bohr, Collected Mathematical Works, Danish Mathematical Society, Copenhagen, 1952.

6. L. Carleson, On convergence and growth of partial sums of Fourier series, Acta Math. 116 (1966), 135-157.

7. F. Carlson, Contribution à la théorie des séries de Dirichlet, Note I, Ark. Mat. Astr. Fys. 16 (1922), 1-19 (nr 18).

8. B. Cole and T. Gamelin, Representing measures and Hardy spaces for the infinite polydisk algebra, Proc. London Math. Soc. 53 (1986), 112-142.

9. I. P. Cornfeld, S. V. Fomin, and Ya. G. Sinai, Ergodic Theory, Grundlehren der mathematischen Wissenschaften 245, Springer-Verlag, New York, 1982.

10. H. M. Edwards, Riemann's Zeta Function, Academic Press, New York, 1974.

11. B. V. Gnedenko, The Theory of Probability, Chelsea Publishing Co, 1968.

12. G. H. Hardy and M. Riesz, The General Theory of Dirichlet's Series, Cambridge University Press, 1915.

13. G. H. Hardy and E. M. Wright, An Introduction to the Theory of Numbers, Third Edition, Oxford University Press, London, 1954.

14. H. Helson, Compact groups with ordered duals, Proc. London Math. Soc. 14A (1965), 144-156.

15. H. Helson, Foundations of the theory of Dirichlet series, Acta Math. 118 (1967), 61-77.

16. H. Helson, Compact groups and Dirichlet series, Arkiv Mat. 8 (1969), 139-143.

17. D. Hilbert, Wesen und Ziele einer Analysis der unendlich vielen unabhängigen Variablen, Rend. Cir. Mat. Palermo 27 (1909), 59-74.

18. J.-P. Kahane, Sur les séries de Dirichlet $\sum_{1}^{\infty} \pm n^{-s}$, C. R. Acad. Sc. Paris 276, A739-A742.

19. E. Landau, Handbuch der Lehre von der Verteilung der Primzahlen, Teubner, Leipzig, 1909, reprinted by Chelsea 1953.

20. W. Schnee, Zum Konvergensproblem der Dirichletschen Reihen, Math. Ann. 66 (1909), 337349.

21. R. M. Young, An Introduction to Nonharmonic Fourier Series, Academic Press, New York, 1980.

Håkan Hedenmalm, Department of Mathematics, Uppsala University, Box 480, S75106 Uppsala, Sweden

E-mail address: haakan@math.uu.se 
E-mail address: lqvist@imf.unit.no

Kristian Seip, Department of Mathematical Sciences, The Norwegian Institute of Technology, N-7034 Trondheim, Norway

E-mail address: seip@imf.unit.no 